ARTICLE

\title{
An immune response characterizes early Alzheimer's disease pathology and subjective cognitive impairment in hydrocephalus biopsies
}

Wenrui Huang 1,2, Anne Marie Bartosch (1) 1,2, Harrison Xiao ${ }^{1,2}$, Suvrajit Maji (1) 1,2, Elliot H. H. Youth (1) ${ }^{2}$, Xena Flowers², Sandra Leskinen ${ }^{2,3}$, Zeljko Tomljanovic (10 ${ }^{2}$, Gail lodice ${ }^{4}$, Deborah Boyett ${ }^{4}$, Eleonora Spinazzi ${ }^{4}$, Vilas Menon (10 2,3, Robert A. McGovern ${ }^{5}$, Guy M. McKhann ${ }^{4,6}$ \& Andrew F. Teich (1) 1,2,3,6凶

Early Alzheimer's disease (AD) pathology can be found in cortical biopsies taken during shunt placement for Normal Pressure Hydrocephalus. This represents an opportunity to study early AD pathology in living patients. Here we report RNA-seq data on 106 cortical biopsies from this patient population. A restricted set of genes correlate with $A D$ pathology in these biopsies, and co-expression network analysis demonstrates an evolution from microglial homeostasis to a disease-associated microglial phenotype in conjunction with increasing $A D$ pathologic burden, along with a subset of additional astrocytic and neuronal genes that accompany these changes. Further analysis demonstrates that these correlations are driven by patients that report mild cognitive symptoms, despite similar levels of biopsy $\beta$-amyloid and tau pathology in comparison to patients who report no cognitive symptoms. Taken together, these findings highlight a restricted set of microglial and non-microglial genes that correlate with early $A D$ pathology in the setting of subjective cognitive decline.

\footnotetext{
${ }^{1}$ Department of Pathology and Cell Biology, Columbia University, New York, NY, USA. ${ }^{2}$ Taub Institute for Research on Alzheimer's Disease and the Aging Brain, Columbia University, New York, NY, USA. ${ }^{3}$ Department of Neurology, Columbia University, New York, NY, USA. ${ }^{4}$ Department of Neurosurgery, Columbia University, New York, NY, USA. ${ }^{5}$ Department of Neurosurgery, University of Minnesota, Minneapolis, MN, USA. ${ }^{6}$ These authors jointly supervised this work: Guy M. McKhann, Andrew F. Teich. ${ }^{凶}$ email: aft25@cumc.columbia.edu
} 
O ngoing work molecularly characterizing Alzheimer's disease $(\mathrm{AD})$ autopsy brain tissue has produced a wealth of information about a wide range of pathophysiologic processes in $\mathrm{AD}^{1-4}$. Less work has been done to molecularly characterize $\mathrm{AD}$ pathology in surgical biopsy tissue from living patients, which is more difficult to obtain but offers unique advantages for studying $\mathrm{AD}$ pathophysiology. For example, multiple groups have studied AD pathology in cortical biopsies from normal pressure hydrocephalus (NPH) patients ${ }^{5-8}$, and insights from this work have furthered our understanding of $\mathrm{AD}$ pathophysiology. Chronic hydrocephalus in the aging population can occur for a variety of reasons, although the etiology is often unclear. In the absence of a clear etiology, most of these cases are categorized as idiopathic NPH (iNPH). Placing a ventricular shunt is often effective for symptom relief in the setting of NPH/ chronic hydrocephalus $7,9,10$, although which patients will have persistent long-term clinical benefit remains to be determined ${ }^{11}$. At the time of shunt placement, a cortical biopsy is often obtained at the brain entry point to look for possible coexistent brain pathology. Perhaps not surprisingly, cortical biopsies taken from elderly NPH patients at shunt placement have been shown to have a relatively high frequency of $\beta$-amyloid plaque pathology, ranging from 42 to $67 \%^{8,12}$, perhaps due to the fact that earlystage $\mathrm{AD}$ in many cases may actually be causing some of the symptoms attributed to NPH/chronic hydrocephalus. Consistent with this hypothesis, the presence of either (1) severe $\beta$-amyloid plaque pathology or (2) a cerebrospinal fluid (CSF) AD signature of high phospho-tau/ $\beta$-amyloid ratio have both been shown to predict a lack of response to shunting ${ }^{8,13}$. Interestingly, unlike $\beta$ amyloid plaque pathology, tau pathology is relatively sparse in $\mathrm{NPH}$ cortical biopsies ${ }^{8}$, although some studies have found trace tau pathology at higher levels ${ }^{14}$, which is consistent with the fact that most patients coming to shunt surgery do not show severe cognitive impairment (those patients who are pre-AD are likely to be at a Braak stage with sparse to no neocortical tangles). These findings are in agreement with the average age at biopsy for NPH patients, which in the low to mid $70 s^{5-7}$. This age range is significantly lower than the average age of existing $\mathrm{AD}$ autopsy cohorts, which are typically in the mid to upper $80 \mathrm{~s}^{1,4}$, and is close to the average age of initial clinical presentation for $\mathrm{AD}$ (75.5 years $)^{15}$. The hypothesis that NPH patients with AD pathology represent a pre-AD group is further buttressed by a recent longitudinal study of $335 \mathrm{NPH}$ patients showing that NPH patients progress to $\mathrm{AD}$ at a higher rate than an aged reference population ${ }^{6}$. This study showed that $\mathrm{AD}$ pathology on brain biopsy ( $\beta$-amyloid and tau) is the single best predictor of progression to $\mathrm{AD}$ in comparison with other clinical and radiographic metrics. Nevertheless, there were patients in this study (in accordance with previous published work ${ }^{16-18}$ ) who have AD pathology and do not progress to AD. It should also be noted that the confounding factor of hydrocephalus is present in any NPH cohort. Thus, all of the above studies of NPH patients as a pre$\mathrm{AD}$ group should be interpreted with these caveats in mind (see "Discussion").

Recent technological advancements in RNA-sequencing (RNAseq) have led to the generation of transcriptomic datasets from post-mortem brain tissue of patients affected by $\mathrm{AD}^{1-4}$. Analysis of this data has shed light on many aspects of AD pathophysiology, such as highlighting the similarities and differences in microglial gene expression between mouse models of $\mathrm{AD}$ and human $\mathrm{AD}$ autopsy tissue ${ }^{19}$, as well as defining microglial subtypes that may be relevant in human $\mathrm{AD}^{20}$. Surgical biopsies from hydrocephalus patients have already proved useful for understanding the clinical consequences of early $\mathrm{AD}$ pathology and may also be useful for understanding early transcriptomic changes in $\mathrm{AD}$. Note that, in addition to the advantages mentioned above, surgical biopsies are also free of gene expression changes that accompany end-of-life hypoxia/apoptotic state, as well as any changes in RNA caused by post-mortem degradation, and all cognitive and clinical data curated from the patients' peri-operative charts are contemporaneous with the time of tissue acquisition. Thus, surgical biopsies from NPH patients represent a valuable opportunity to examine the transcriptomic profile of brain tissue in living patients with early $\mathrm{AD}$ pathology.

In this work, we report RNA-seq data from $106 \mathrm{NPH}$ cortical biopsies and correlate changes in gene expression with co-morbid $\mathrm{AD}$ pathology in this patient population. Analysis of these biopsies shows a restricted set of microglial and non-microglial genes that correlate with histological measurements of $\beta$-amyloid and tau pathology primarily in patients who report subjective cognitive impairment. Specifically, we identify a gene expression module enriched for murine disease-associated genes that positively correlates with $\mathrm{AD}$ pathology and a module enriched for murine homeostatic genes that negatively correlates with $\mathrm{AD}$ pathology, and in aggregate, this is more consistent with the existing mouse literature than other publicly available $\mathrm{AD}$ autopsy datasets $1,2,4,19,21,22$. Finally, these microglial modules are also correlated with microglial plaque association and changes in microglial morphology, and this change is not sensitive to cognitive status. Taken together, these data suggest that an initial microglial response to $\mathrm{AD}$ pathology is associated with accumulating pathology, non-microglial cell responses, and patientreported cognitive status.

\section{Results}

In this study, we examine changes in gene expression that accompany early $\mathrm{AD}$ pathology in cortical biopsies that were removed in the course of shunt placement for NPH and compare the results with $\mathrm{AD}$ pathology on histology and contemporaneously gathered cognitive data (see Fig. 1 for our workflow and "Methods" for further details on our cohort). Specifically, we performed RNA-seq on 106 biopsies from NPH patients, with an average age of 74.9 years. In all cases, patients were shunted for chronic hydrocephalus by the same surgical team, and biopsies were taken from a specific area of frontal cortex (2/3 of our cohort) or parietal cortex (1/3 of our cohort). The decision to shunt/biopsy in frontal or parietal cortex was made by the surgeon based on cosmetic considerations (see "Methods"). Changes in gene expression that correlate with $\mathrm{AD}$ pathology in our samples trend similarly in frontal and parietal cortex (see Supplementary Data 1), and even when we combine all samples, very few individual genes reach statistical threshold (see below).

Immune/microglia-specific genes are strongly correlated with NPH AD pathology. We initially processed our RNA-seq data by regressing out variability in gene expression not associated with our primary variables of interest $(\beta$-amyloid plaque and tau pathology ${ }^{23}$. $\beta$-Amyloid plaques were counted per square millimeter area on slides of tissue immunostained with 6E10 antibody. In order to quantify tau, we devised a rating scale to grade the minimal degree of tau pathology seen in NPH biopsy slides immunostained with AT8 antibody (see Supplementary Fig. 1 for examples of each grade). Grade 0 was given to biopsies with no tau pathology $(n=42)$. Grade 1 was given to biopsies that have any tau pathology at all, usually one or more dystrophic neurites $(n=39)$. Grade 2 was given to biopsies that have at least one taupositive neuron or neuritic plaque $(n=18)$. Grade 3 was reserved for biopsies with tau pathology evenly distributed throughout the biopsy $(n=7)$. 


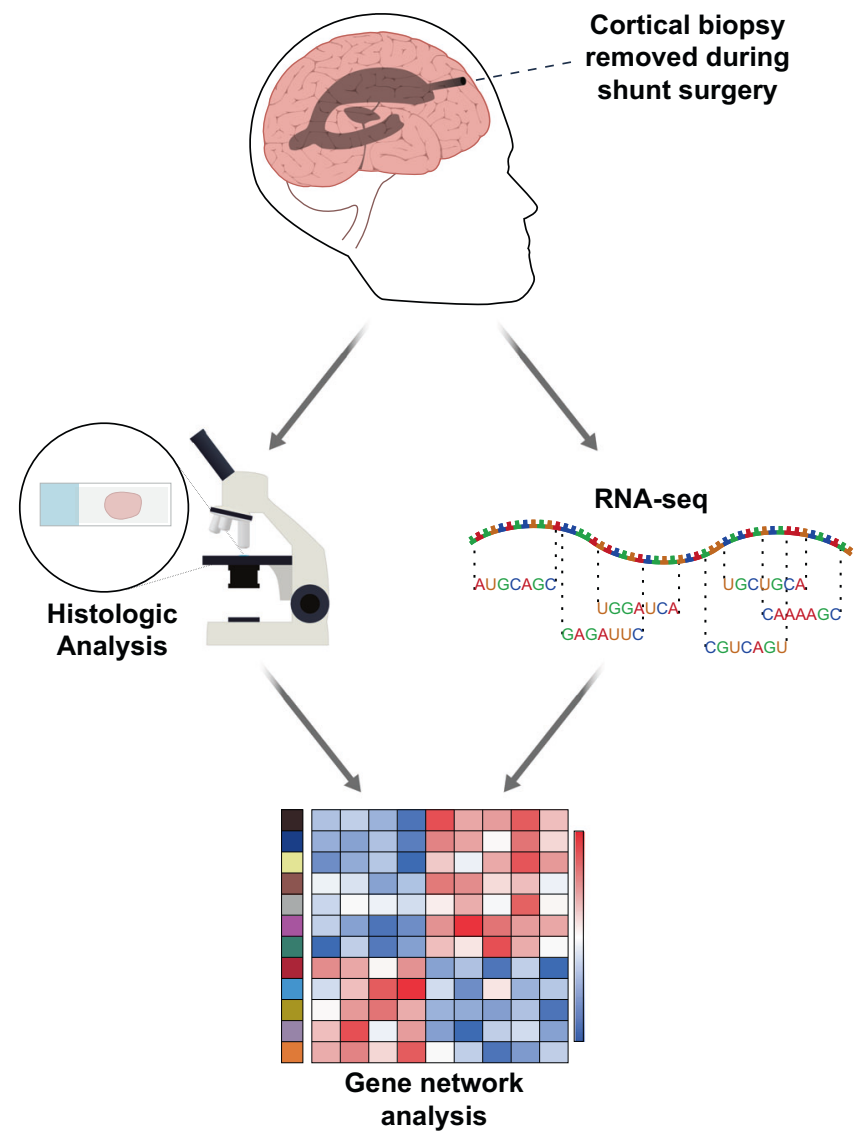

Fig. 1 Biopsies removed for ventricular shunting in the operating room are immediately split in half. Half of the biopsy is frozen in liquid nitrogen and sent for RNA-seq. The other half is formalin fixed and paraffin embedded for subsequent pathologic analysis (see "Methods").

Initial analysis of the data identified a restricted set of 38 genes that passed false discovery rate (FDR) of 0.1 at the individual gene level that correlated with either $\beta$-amyloid and/or tau burden. Indeed, one of the most striking things about our initial analysis is the overall consistency of gene expression signatures in these biopsies, especially given the large-scale changes in gene expression seen in many other autopsy-based cohorts that include brains with clinical $\mathrm{AD}^{1-4}$. Note that our sample size of 106 is less than several well-known autopsy cohorts ${ }^{1,4}$. While sample size could contribute to the lower number of significant genes in this study, it should also be noted that the correlations in this study are calculated somewhat differently from correlations reported in previous studies of AD autopsy tissue ${ }^{1,4}$. Specifically, we are correlating changes in gene expression to $\mathrm{AD}$ pathology quantified in an immediately adjacent piece of tissue. In contrast, other datasets usually correlate gene expression in a single piece of tissue with global metrics of $\mathrm{AD}$ pathology or with $\mathrm{AD}$ pathology in tissue that is not necessarily contiguous with the tissue used for RNA-seq, all of which might lead to weaker correlations with RNA-seq data. Thus, while our lower sample size could contribute to lower power in comparison to some studies, we are likely to see stronger correlations with pathology due to our study design. In order to further test the overall consistency of gene expression in these biopsies, we performed differential gene expression and directly compared biopsy tissue with no $\mathrm{AD}$ pathology (no $\beta$-amyloid or tau, $n=32$ ) to biopsy tissue with any AD pathology (either $\beta$-amyloid and/or tau, $n=74)$. This analysis only yielded two genes that passed FDR of 0.1 (Supplementary Data 1). Similar analyses based only on $\beta$-amyloid (no $\beta$-amyloid, $n=49$ vs. any $\beta$-amyloid, $n=57$ ) or tau (no tau, $n=42$ vs. any tau, $n=64$ ) yielded 19 and 4 genes passing FDR 0.1 respectively, consistent with the overall low number of significant genes in our correlation analysis (Supplementary Data 1).

The genes that pass FDR threshold in our NPH data are enriched for immune response genes, many of which have been tied to AD. Figure 2 shows the top 20 genes that correlate with $\beta$ amyloid plaques and tau burden. A number of immune- or microglia-specific genes were among the top 20 list, including TREM 2 and C4B, both of which have been implicated in the immune response in $\mathrm{AD}^{24,25}$. These results point to microglia/ immune response changes as being important in the very earliest stages of $\mathrm{AD}$ pathology and occur before other physiologic changes appear at the bulk RNA-seq level. To confirm this, we determined the level of overlap between our list of genes and lists of genes that characterize specific cell types using human singlenucleus RNA-seq data ${ }^{26}$. A Fisher's exact test (FET) confirmed that microglia-specific genes are uniquely overrepresented among the genes that individually passed our FDR threshold (Fig. $2 \mathrm{c}$ and Supplementary Data 1). It should also be noted that several of the individual genes that reach significance in our analysis are astrocytic. For example, glial fibrillary acidic protein (GFAP) is a reactive astrocyte marker, and CD44, SERPINA3, and C4B have all been associated with disease-associated astrocytes ${ }^{19,27,28}$. While microglia are the predominant cell type represented in our transcriptomic data, an astrocytic response is also clearly present at this early stage of $\mathrm{AD}$ pathology.

AD pathology gene correlations are strongest in patients with subjective cognitive impairment. Previous work with AD RNAseq tissue has used gene network analysis to further clarify how groups of genes correlate with various $\mathrm{AD}$ traits ${ }^{1}$. When we performed weighted gene co-expression network analysis (WGCNA) ${ }^{29}$ on our NPH data, we identified in total 58 gene coexpression modules, only 3 of which are significantly correlated with either $\beta$-amyloid or tau burden (saddlebrown, orange, and darkgrey modules-Fig. 3; see Supplementary Data 2 for full results of WGCNA analysis), consistent with analysis at the single-gene level that a restricted set of genes correlate with $\mathrm{AD}$ pathologic traits in this cohort. To further refine our analysis, patient charts were curated for data that would help differentiate the patients by cognitive status. Although rigorous cognitive testing was not consistently carried out, the majority of patients and their families were asked whether they had experienced subjective cognitive impairment during an exam close in time to the biopsy date (see "Methods"). Using this simple metric (yes vs. no), we were able to assign 93 of our sequenced biopsies into these two groups, with 59 replying yes and 34 replying no (the remaining 13 biopsies came from patients where we were unable to locate the answer to this question in the chart; see Supplementary Table 1 for distribution of samples by cognitive status). Patients who reported subjective cognitive impairment had non-significantly higher $\beta$-amyloid and tau load than patients who reported no cognitive impairment ( $p$ value for $\beta$-amyloid $=0.21 ; p$ value for tau $=0.66$ by Mann-Whitney $U$ test). Interestingly, all of the tau grade 3 biopsies with cognitive information have a history of subjective cognitive complaint. However, these biopsies are so few in number (7) that they do not significantly affect the overall analysis. Interestingly, there is significantly less co-occurrence of $\beta$-amyloid pathology and tau pathology in the same biopsy from patients who report no cognitive impairment when compared to patients who do report subjective cognitive impairment (see Supplementary Fig. 2). This suggests that $\mathrm{AD}$ pathology may be more widespread in 


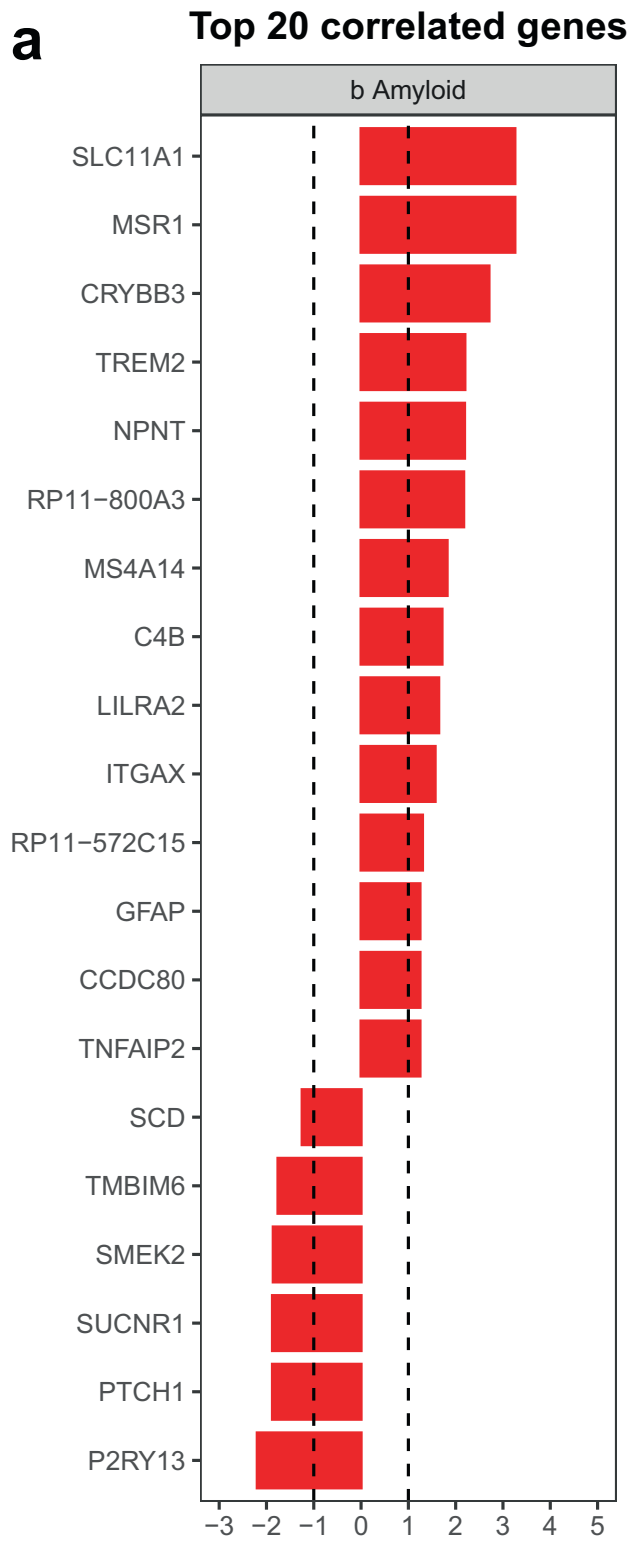

signed negative $\log 10$ adj. $p$ val b

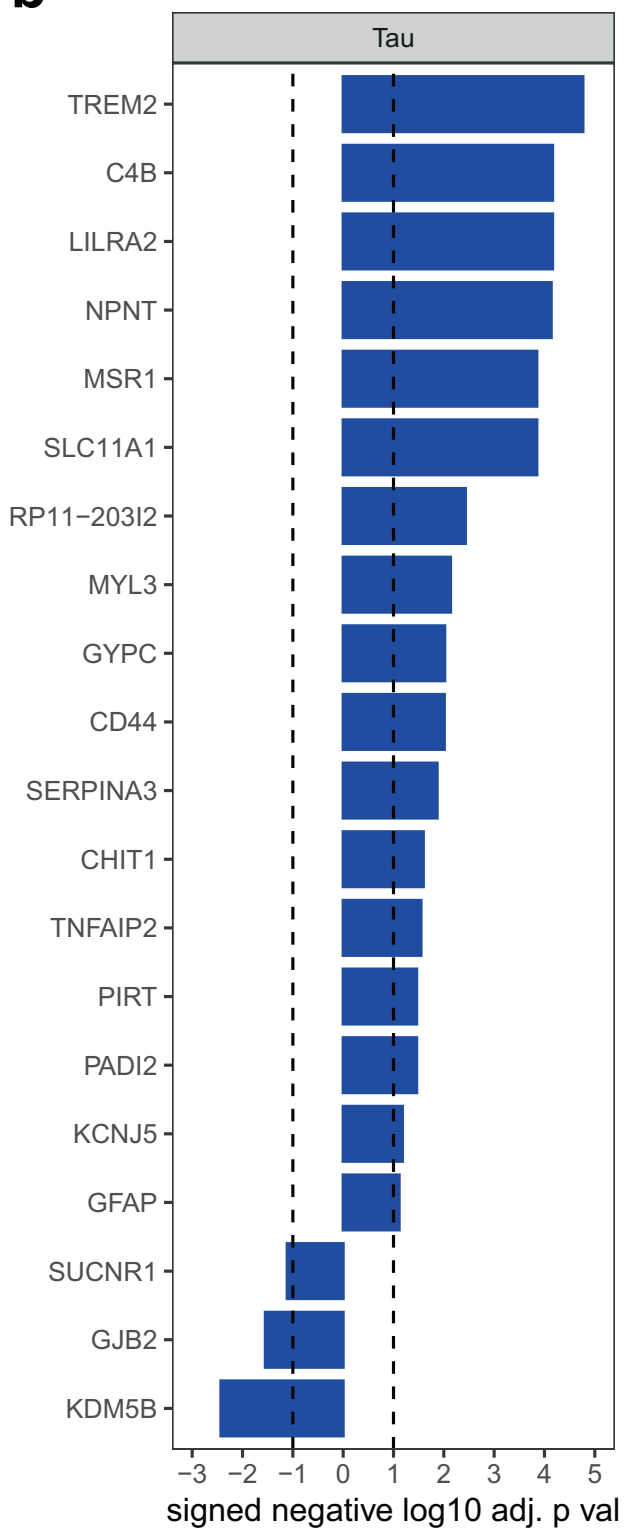

\section{Top 20 correlated genes}

C

Cell type enrichment analysis

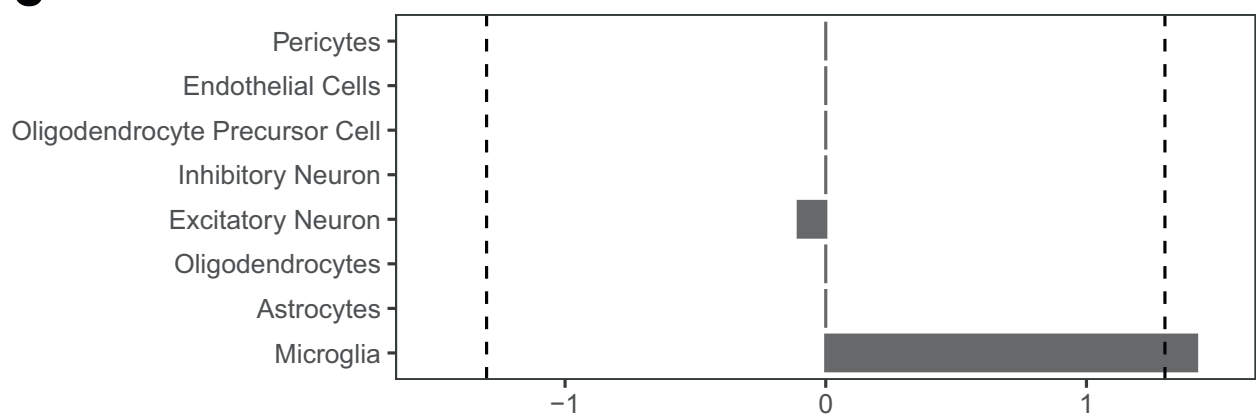

signed negative $\log 10$ adjusted $p$ value

Fig. 2 A restricted set of genes correlate with AD pathology in NPH biopsies. Shown are the top 20 genes that correlate with $\beta$-amyloid (a) and tau (b) burden in NPH biopsies (FDR adjusted using the Benjamini-Hochberg procedure across all genes in the transcriptome-dotted line is FDR $=0.1$; see Supplementary Data 1 for the full list; all correlations are Spearman's correlations with two-sided significance). c A two-sided Fisher's exact test confirmed that microglia-specific genes are overrepresented among the genes that individually passed our FDR threshold using human single-nucleus RNA-seq data21 (c shows Bonferroni adjusted $p$ values; dotted line $=p$ value of 0.05; See Supplementary Data 1 for numerical values). 


\section{a WGCNA modules of interest versus b Amyloid correlation}

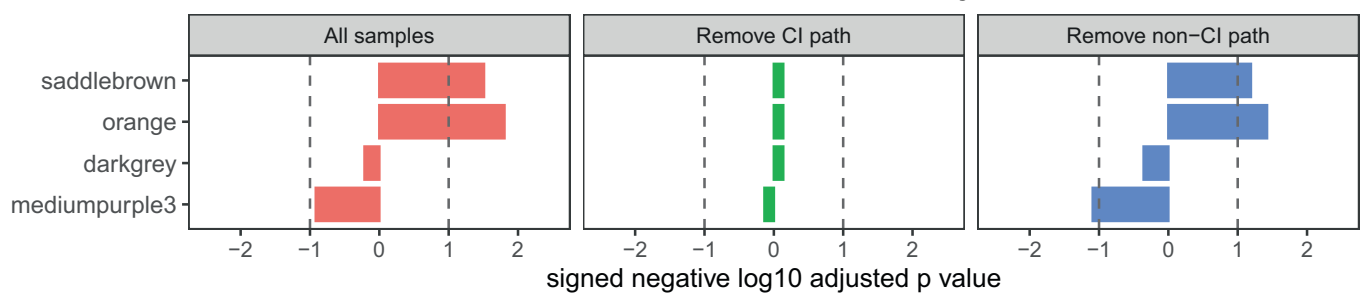

All samples

Remove $\mathrm{Cl}$ path

Remove non- $\mathrm{Cl}$ path

b

WGCNA modules of interest versus Tau correlation
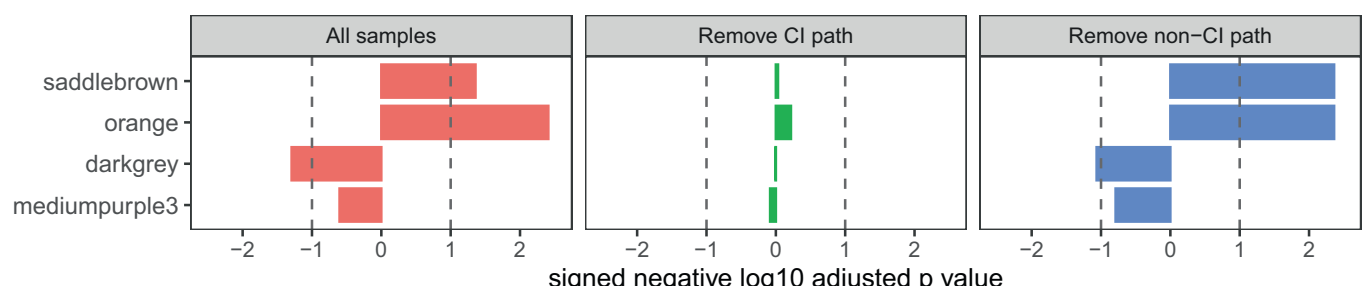

All samples

Remove Cl path

Remove non-Cl path

C

Cell type Spearman correlation with Bonf adjusted $p$ values

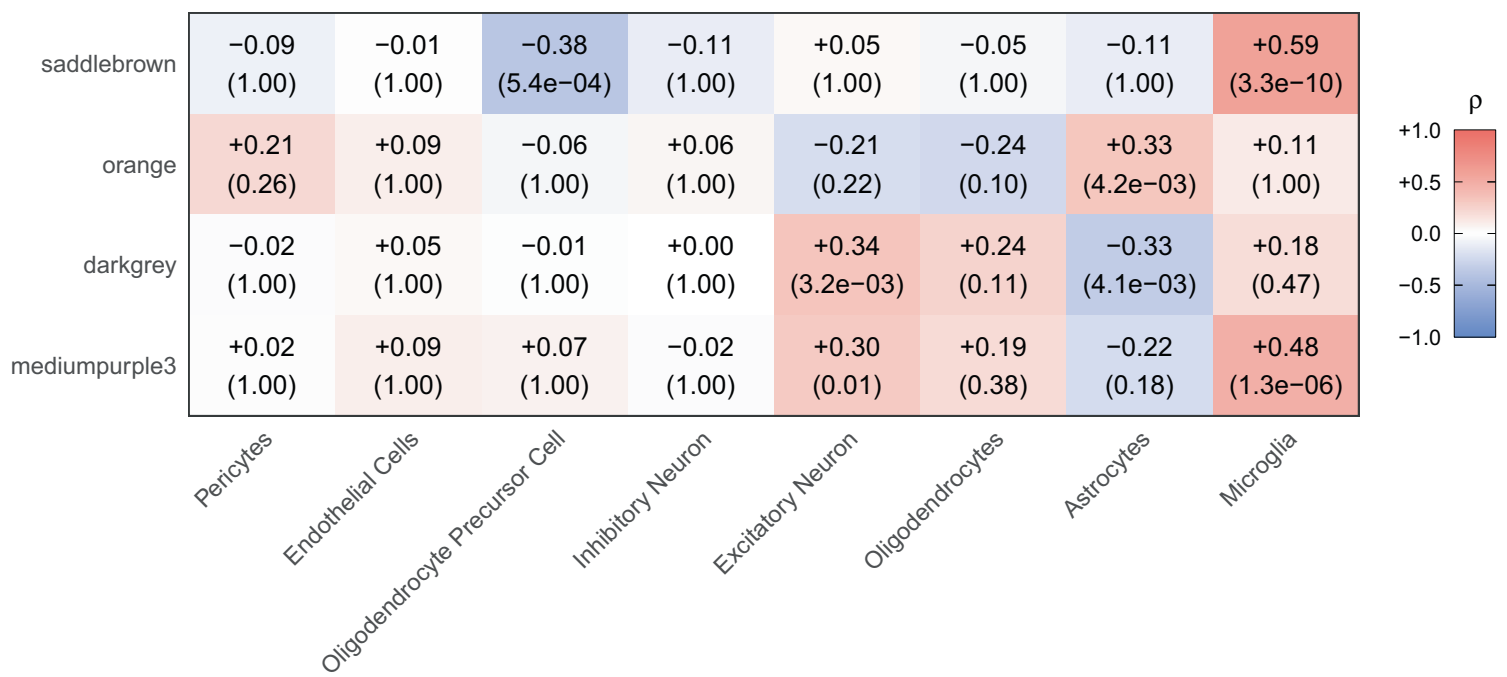

Fig. 3 Gene expression modules correlate with AD pathology in the setting of subjective cognitive impairment. a, $\mathbf{b}$ Consistent with the single-gene analysis, WGCNA shows that only three modules (saddlebrown, orange, and darkgrey) correlate significantly with $\beta$-amyloid (a) and tau (b) when all samples $(n=106)$ are considered (red bars on left; see Supplementary Data 2 for the correlations for all WGCNA modules from this analysis and Supplementary Data 3 for the list of genes in key modules in this paper). Subjective cognitive status strongly influences how microglial modules correlate with $\beta$-amyloid and tau pathology (green and blue bars). When we removed all samples with AD pathology from our cohort that reported subjective cognitive impairment (leaving samples with AD pathology without subjective cognitive impairment and all non-pathology specimens; the resulting $n=66$ ), this abolished the significant correlation of the modules with AD pathology (green bars). In contrast, when we do the converse (i.e., remove all samples with $A D$ pathology from our cohort that do not report subjective cognitive impairment, leaving samples with AD pathology with subjective cognitive

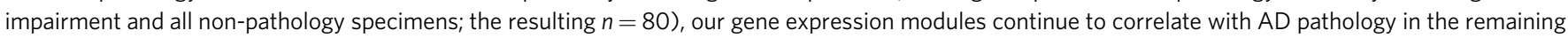
biopsies (blue bars). In fact, one module that was previously not significant becomes significant (mediumpurple3). a, b are FDR adjusted using the Benjamini-Hochberg procedure across all 58 modules in our WGCNA analysis-dotted line in $\mathbf{a}$, b is FDR =0.1; all correlations are Spearman's correlations with two-sided significance. See Supplementary Table 1 for a full breakdown of the cognitive status of all samples in our cohort. c The modules in $\mathbf{a}$, $\mathbf{b}$ were correlated with cell-type-specific gene lists (using human single-nucleus RNA-seq data ${ }^{21}$ ). While saddlebrown and mediumpurple3 are clearly microglial, darkgrey and orange are more weakly neuronal and astrocytic, respectively, by Spearman's correlation (each row is separately Bonferroni adjusted, with adjusted two-sided $p$ values in parentheses below Spearman's correlations; see Supplementary Fig. 3 for Fisher's exact test enrichment of these modules with cell-type-specific gene lists and Supplementary Data 5 for all Spearman's correlations and enrichment values and $p$ values for these modules with different cell types).

patients who report cognitive impairment (so that we are more likely to see both $\beta$-amyloid and tau in a small biopsy), even if the local density of $\mathrm{AD}$ pathology in these biopsies is not significantly higher compared to patients who report no cognitive impairment.
Although cognitive status does not predict significantly higher local density of AD pathology in biopsies in our cohort, we did find that the correlations of our modules with AD pathology are being driven by patients who report cognitive impairment. When we removed all samples from individuals with $\mathrm{AD}$ pathology who 
reported cognitive impairment, this abolished the significant correlation of the modules with $\mathrm{AD}$ pathology in the remaining biopsies (Fig. 3a, b; see Supplementary Data 2 for full analysis with all WGCNA modules). In contrast, when we do the converse (i.e., remove samples from individuals with $\mathrm{AD}$ pathology who did not report cognitive impairment), our gene expression modules continued to correlate with $\mathrm{AD}$ pathology in the remaining biopsies. In fact, an additional module reached the significance threshold (mediumpurple3; see Supplementary Data 3 for genes in the modules of interest in this manuscript). To further examine the sensitivity of gene correlations with $\mathrm{AD}$ pathology to cognitive status, we ran 1000 iterations where we randomly replaced half of the samples with $\mathrm{AD}$ pathology in the analysis shown in blue (Fig. 3a, b) with pathology samples in the analysis shown in green (i.e., pathology samples with subjective cognitive impairment are being randomly replaced with pathology samples without documented cognitive impairment). As noted in Supplementary Data 4 , this did not statistically change the overall distribution of the burden of pathology in any of the simulations. In contrast, all 4 of our modules fail to pass 0.1 FDR significance in their correlation with $\beta$-amyloid and tau for the majority of the simulations. Taken together, these findings indicate that the correlations of these modules with $\mathrm{AD}$ pathology are highly sensitive to cognitive status.

In an effort to better characterize these modules, we first determined whether these modules correlate with cell-typespecific gene lists. Analysis with gene lists from human singlenucleus RNA-seq data ${ }^{21}$ identified specific cell class assignments for our modules (Fig. 3c), and this is further supported by enrichment analysis (Supplementary Fig. 3 and Supplementary Data 5). These analyses support the view that the saddlebrown and mediumpurple3 modules are predominantly microglial. While the data is somewhat more mixed for darkgrey and orange, the overall trend is that darkgrey is neuronal while orange is astrocytic, which is broadly consistent with the positive correlation of the orange module and negative correlation of the darkgrey module with $\beta$-amyloid and tau in the NPH data. Ontology analysis is consistent with these observations (Fig. 4; see Supplementary Data 6 for full ontology analysis results), with saddlebrown and mediumpurple 3 characterized by immune response ontology gene sets and darkgrey by neuronal gene sets (note that the orange module's cell-type specificity is less clear from ontology analysis).

In our implementation of WGCNA, we allowed for both positive and negative gene correlations within modules (unsigned implementation; see "Methods"). This allowed us to capture more complex changes in physiology within the same module. In addition, using unsigned modules also eliminates the extra analysis step needed to pair signed modules that are anticorrelated with each other. Of the four modules that pass FDR threshold in Fig. 3, darkgrey is notable for having the largest fraction of genes that negatively correlate with the PC1 eigengene (37\% of darkgrey genes correlate negatively with the PC1 eigengene, while the other three modules all have $<20 \%$ of their genes negatively correlating with PC1; see Supplementary Data 3). For all four of our modules of interest, ontology analysis of positively correlating genes produced similar gene sets as the ontology analysis of the full module (Supplementary Data 6), as well as similar levels of correlations and enrichment with the full module (Supplementary Data 5), consistent with the positively correlating genes dominating the signature of these modules. On the other hand, ontology analysis of negatively correlating genes revealed no significant gene sets for saddlebrown, one gene set for mediumpurple3, and several gene sets without a clear theme for orange. In contrast, ontology analysis for negatively correlating genes in darkgrey revealed several significant gene sets related to lipid metabolism (including lipid binding and lipid transporter activity). This suggests that upregulation of lipid metabolism may be an early change that occurs in tandem with early neuronal dysfunction and loss of synaptic/neuronal genes in AD. Lipid metabolism is increasingly recognized as playing an important role in $\mathrm{AD}$ pathogenesis ${ }^{30,31}$, and two of the genes from these ontology gene sets (ApoB and PCTP) have recently been implicated in $\mathrm{AD}$ through analysis of genome-wide association study data ${ }^{32,33}$. In addition to these changes, we also note several other compensatory genes in darkgrey that negatively correlate with the PC1 eigengene, such as HSB1 and neuroglobin, which have both been shown to increase in $\mathrm{AD}$ and are thought to be part of the stress response $\mathrm{s}^{34-36}$. In summary, the darkgrey module suggests that we may be capturing early neuronal changes along with compensatory/reactive changes in these biopsies that correlate with increasing pathology most significantly in patients with subjective cognitive impairment. Moreover, this analysis suggests that, while at the single-gene level the changes we are observing are overwhelmingly microglial (Fig. 2), WGCNA is identifying correlating genes from other cell types that may be less significant at the single-gene level.

NPH modules can be found in other publicly available AD datasets and are enriched for previously identified sets of homeostatic and disease-associated microglial response genes. We next determined how applicable our findings in NPH tissue are for brains with diagnosed AD. The Religious Orders Study and Memory and Aging Project (ROSMAP) dataset constitutes one of the largest datasets of RNA-seq data from AD autopsy neocortex, and so we sought to examine how well our modules from the NPH tissue correlate with pathologic stigmata of AD in this cohort. RNA-seq and associated metadata for ROSMAP ${ }^{1}$ was downloaded from the AMP-AD Knowledge Portal, and we first regressed out variability in gene expression not correlated with disease-relevant metadata, similar to our processing pipeline for NPH data (see "Methods"). We first sought to determine whether the modules that correlate with $\beta$-amyloid and tau in our NPH data correlate with CERAD, Braak, or MMSE score in the ROSMAP data. As shown in Fig. 5a, saddlebrown and orange both have significant positive correlations with CERAD and Braak stage from ROSMAP, while darkgrey negatively correlates with these metadata. In addition, saddlebrown and orange negatively correlate with MMSE and darkgrey positively correlates with MMSE in the ROSMAP data, suggesting that these modules may also be related to cognitive decline (see Supplementary Data 8 for correlations and $p$ values). In summary, our glial modules are positively correlating with pathology and cognitive decline while our neuronal module is correlating in the opposing direction, all of which is consistent with known transcriptional changes in the AD RNA-seq literature ${ }^{1,19,21}$. We also examined an additional dataset of frontal cortex RNA-seq data generated at Mount Sinai (MSSM) ${ }^{4}$ and found a similar trend to the ROSMAP data (Fig. 5b).

Strikingly absent from both the ROSMAP and MSSM data is any correlation of mediumpurple 3 with $\mathrm{AD}$ pathology or cognition. The mediumpurple 3 is one of the two microglial modules highlighted in Fig. 3, the other being saddlebrown. Note that, in our NPH data, the saddlebrown module positively correlates with $\mathrm{AD}$ pathology, whereas the mediumpurple3 module negatively correlates with $\mathrm{AD}$ pathology. In an effort to characterize these modules further and explain discrepancies with the autopsy-based cohorts, we first examined which of the modules previously identified in the ROSMAP data most overlap with the modules in our NPH data. Interestingly, both mediumpurple 3 and saddlebrown overlap the most with the same 
a

\begin{tabular}{|c|c|}
\hline Top 10 ontology (saddlebrown) & q value \\
\hline $\begin{array}{c}\text { antigen processing and presentation } \\
\text { antigen processing and presentation of peptide antigen }\end{array}$ & $2.30 \mathrm{e}-13$ \\
\hline $\begin{array}{r}\text { antigen processing and presentation of peptide via MHC class II } \\
\text { lumenal side of endoplasmic reticulum membrane }\end{array}$ & $2.47 \mathrm{e}-13$ \\
\hline antigen processing and presentation of exogenous antigen & $9.55 e-13$ \\
\hline immune response & $1.92 e-11$ \\
\hline regulation of immune system process & $3.41 e-11$ \\
\hline endocytic vesicle membrane & $7.44 e-11$ \\
\hline vesicle membrane & $1.38 e-10$ \\
\hline immune response-regulating signaling pathway & $6.52 e-09$ \\
\hline
\end{tabular}

C

\begin{tabular}{|c|c|}
\hline Top 10 ontology (darkgrey) & q value \\
\hline neuron part & $1.44 \mathrm{e}-05$ \\
\hline synaptic signaling & $6.00 \mathrm{e}-04$ \\
\hline somatodendritic compartment & $1.72 \mathrm{e}-03$ \\
\hline neuronal cell body & $6.81 \mathrm{e}-03$ \\
\hline phagocytic cup & $1.48 \mathrm{e}-02$ \\
\hline regulation of biological quality & $1.53 \mathrm{e}-02$ \\
\hline cell body & $1.84 \mathrm{e}-02$ \\
\hline cell projection & $1.84 \mathrm{e}-02$ \\
\hline cell projection part & $1.84 \mathrm{e}-02$ \\
\hline plasma membrane bounded cell projection part & $2.21 \mathrm{e}-02$ \\
\hline
\end{tabular}

b

\begin{tabular}{|c|c|}
\hline Top 10 ontology (orange) & q value \\
\hline collagen-containing extracellular matrix & $1.51 e-05$ \\
\hline extracellular matrix & $8.00 e-05$ \\
\hline tube development & $1.80 e-04$ \\
\hline chitin binding & $9.69 e-04$ \\
\hline angiogenesis & $2.29 e-03$ \\
\hline tube morphogenesis & $2.29 e-03$ \\
\hline extracellular space & $3.06 e-03$ \\
\hline extracellular structure organization & $5.09 e-03$ \\
\hline blood vessel development & $6.11 e-03$ \\
\hline apical part of cell & $1.83 e-02$ \\
\hline
\end{tabular}

d

Fig. 4 Module ontology analysis suggests cell type specificity. The top 10 ontology groups for saddlebrown (a), orange (b), darkgrey (c), and mediumpurple3 (d) are displayed, along with an FDR-adjusted $q$ value that is based on all ontology group one-sided Fisher's exact test $p$ values that were tested (see Supplementary Data 6 for full ontology analysis for these four modules, including all $p$ values and FDR-adjusted $q$ values).

module from Mostafavi et al. ${ }^{1}$; as seen in Fig. $5 c$, d, this is module 116 (see Supplementary Data 9 for full analysis of the overlap of our four NPH modules of interest with modules from Mostafavi et al. $\left.^{1}\right)$. Mostafavi et al. identified module 116 as a general microglial module ${ }^{1}$, and the fact that both saddlebrown and mediumpurple 3 overlap primarily with 116 suggests that microglial genes are correlating with one another differently in our data than in ROSMAP.

Noting that saddlebrown increases with $\mathrm{AD}$ pathology and mediumpurple 3 decreases with $\mathrm{AD}$ pathology in the NPH data, we next determined whether the saddlebrown and mediumpurple 3 modules were associated with known sets of disease-associated microglial genes. Specifically, two recent studies (Keren-Shaul et al. $^{37}$ and Mathys et al. ${ }^{38}$ ) have identified a transition from homeostasis to a late-stage/disease-associated microglial phenotype in $\mathrm{AD}$ mouse models, suggesting that, as $\mathrm{AD}$ pathology accumulates, microglia undergo a shift in their transcriptomic profile. As part of this analysis, we generated a list of the top five microglial modules from our WGCNA analysis (based on enrichment with human microglial genes from Mathys et al. ${ }^{21}$ ) and compared the relative enrichment of these five modules for different disease-stage gene groups from Keren-Shaul et al. ${ }^{37}$ and Mathys et al. ${ }^{38}$ (see Supplementary Data 13 for overlap of these modules with lists from Keren-Shaul et al. ${ }^{37}$ and Mathys et al. ${ }^{38}$ ). We then compared this analysis with the distribution of different disease-stage gene groups in the top five microglial modules from the ROSMAP analysis ${ }^{39}$. As seen in Fig. 5, microglial modules from our NPH study largely segregate homeostatic and diseaseassociated gene groups, with no module showing simultaneous enrichment for homeostatic, early-stage, and late-stage genes (and only one module, salmon4, showing both homeostatic and late-stage enrichment). Interestingly, the mediumpurple3 module significantly overlaps with the homeostatic gene lists from these mouse papers. In contrast, the saddlebrown module overlaps exclusively with DAM stage 2 and late response genes from these papers. The corresponding negative and positive relationship of these same two modules with AD pathology in the NPH data suggests that these two modules are at least partially tracking an early evolution from homeostatic to late-stage/disease-associated microglia in biopsies with early $\mathrm{AD}$ pathology that was first documented in mice.

In contrast, the five microglial modules from Mostafavi et al. are enriched for a broader range of gene lists identified in the mouse literature, and four out of the five are enriched with a list from every category (i.e., homeostasis, early stage, and late stage; Fig. 5j-n; see Supplementary Data 10 for all enrichment values and $p$ values). Interestingly, none of the microglial modules from Mostafavi et al. negatively correlate with $\mathrm{AD}$ pathology ${ }^{1}$, in contrast to our homeostatic module mediumpurple3. We also examined whether the mouse gene groups themselves correlated with AD pathology in NPH, ROSMAP, and MSSM. As seen in Supplementary Fig. 4 and Supplementary Data 11, the homeostatic gene groups defined in mice trend negatively with pathology in NPH data and are either near zero or positively correlate with $\mathrm{AD}$ pathology in $\mathrm{AD}$ autopsy data. This is again consistent with the finding that homeostatic genes negatively correlate with $\mathrm{AD}$ pathology in $\mathrm{NPH}$ biopsies and have a more complex relationship with pathology in AD autopsy tissue.

Although the microglial response in mice differs from humans in many important ways, this data suggests that the transition from homeostasis to a disease-associated phenotype is also occurring to some extent in the NPH data, whereas many of these 


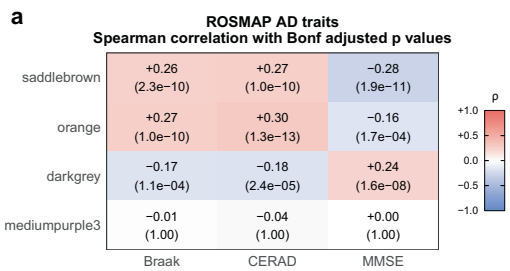

b

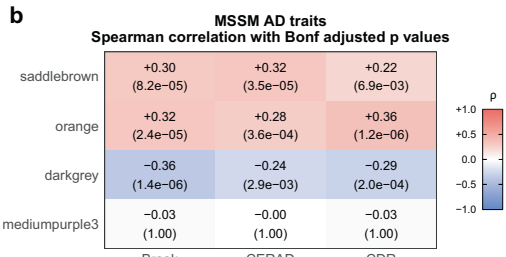

c

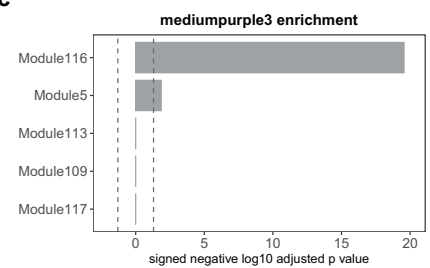

d

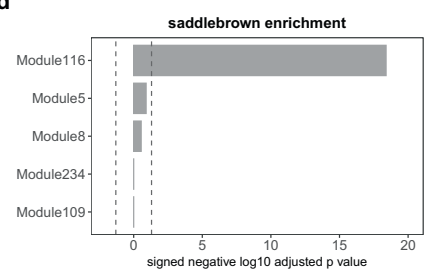

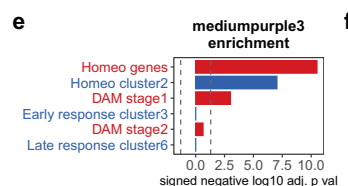

j

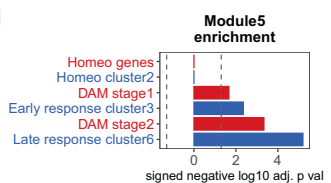

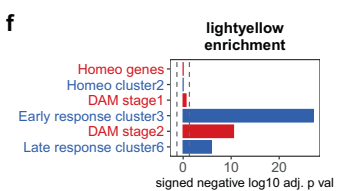

$\mathbf{k}$

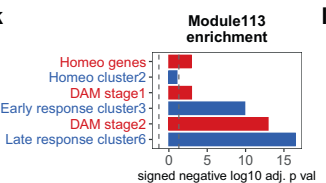

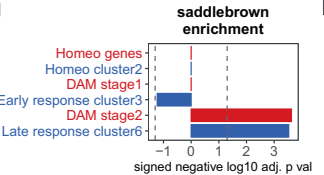

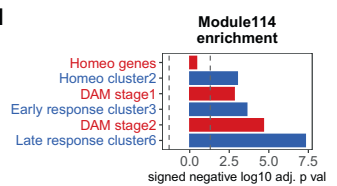

h
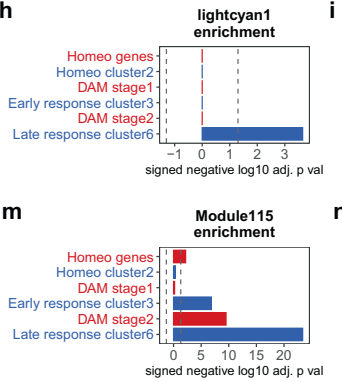
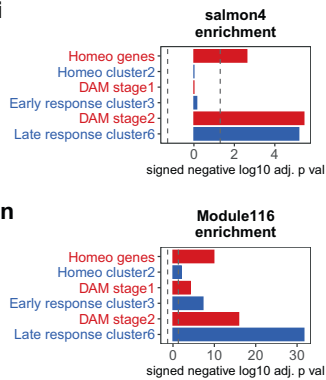

Fig. 5 The modules identified in our NPH data also correlate with AD pathology in autopsy cohorts. a Saddlebrown, orange, and darkgrey correlate with CERAD score, Braak stage, and MMSE score in 596 RNA-seq profiles from the Religious Orders Study and Memory and Aging Project (ROSMAP) Study (representing a range of $A D$ pathologic states) ${ }^{1}$. b Similarly, these modules correlate with CERAD, Braak, and CDR score in RNA-seq profiles from frontal cortex in 183 RNA-seq profiles from the MSSM dataset ${ }^{4}$ (all correlations in $\mathbf{a}, \mathbf{b}$ are Spearman's correlations with two-sided significance). c, d Saddlebrown and mediumpurple3 are both primarily enriched for genes from the same microglial module from Mostafavi et al. ${ }^{1}$, suggesting that microglial genes in our data are correlating differently than in autopsy cohorts (see Supplementary Data 9 for enrichment analysis with two-sided Fisher's exact test $p$ values of saddlebrown, orange, darkgrey, and mediumpurple3 with all modules from Mostafavi et al. ${ }^{1}$ ). e-n The distribution of enrichment for mouse microglial gene lists from Keren-Shaul et al. ${ }^{37}$ (red bars) and Mathys et al. ${ }^{38}$ (blue bars) is more segregated in the top five microglial modules from the NPH data (e-i) than in the top five microglial modules from Mostafavi et al.1,39 (j-n) (see text for details and discussion and Supplementary Data 8-10 for all Spearman's correlations and two-sided Fisher's exact test enrichment $p$ values from these panels). For $\mathbf{a}$, $\mathbf{b}$, each row is separately Bonferroni adjusted, with adjusted $p$ values in parentheses below Spearman's correlations; for two-sided Fisher's exact test in c-n, each panel is separately Bonferroni adjusted-dotted line in $\mathbf{c}-\mathbf{n}$ is $p$ value $=0.05$.

genes are co-correlating in autopsy data. To further explore this phenomenon, we analyzed single-nucleus RNA-seq data from three different $\mathrm{AD}$ tissue studies (Fig. 6 and Supplementary Data 7). Mathys et al. $^{21}$ and Zhou et al. ${ }^{19}$ both analyzed neocortical tissue from $\mathrm{AD}$ vs. control. Both studies found that genes that are increasing in AD overlap with both of our NPH microglial modules, as well as several mouse gene lists at a variety of stages (including both homeostatic and DAM2). This is consistent with both homeostatic and late-stage DAM genes increasing in $\mathrm{AD}$ autopsy tissue, similarly to the ROSMAP bulk RNA-seq data (Supplementary Fig. 4). Note that Mathys et al. also had a control compared to early AD pathology analysis, but genes changing in this comparison do not overlap significantly with either the mediumpurple3 module or either of the two homeostatic mouse lists (Supplementary Data 7), suggesting that this particular comparison is still not capturing the early loss of homeostatic genes seen in the NPH biopsies or the mouse literature (see "Discussion"). Grubman et al. performed singlenucleus RNA-seq on entorhinal cortex samples from $\mathrm{AD}$ vs. control, and this paper shows that a significant subset of homeostatic genes decline in $\mathrm{AD}$ (Fig. 6), which suggests that the autopsy literature is not universally the opposite of the mouse literature with regard to homeostatic genes and $\mathrm{AD}$ pathology. It is also interesting to note that late-stage/DAM2 genes are both increasing and decreasing in $\mathrm{AD}$ in the single-nucleus RNA-seq literature. Although there are a variety of reasons this may be happening, it has also recently been shown that DAM genes in particular are not well represented in single-nucleus RNA-seq data $^{40}$. Indeed, this could also be a reason why the saddlebrown module has weaker associations with the single-nucleus data than the mediumpurple3 module. We further discuss the Thrupp et al. analysis in the context of the single-nucleus RNA-seq analysis presented here in the "Discussion."

Single whole-cell sequencing of microglia presumably circumvents the problems identified by Thrupp et al., and we compared our modules to two recent single-cell studies to further examine the applicability of our findings to the $\mathrm{AD}$ autopsy literature. Olah et al. recently identified 9 distinct microglial clusters from a set of $\mathrm{AD}$ and non-AD tissue ${ }^{20}$, and the two most relevant clusters for our findings are shown in Fig. 7 (see Supplementary Data 7 for analysis with all clusters from this paper $)^{20}$. Cluster 2 is the cluster most enriched for genes from homeostatic groups, which Olah et al. also defined as a homeostatic group using a Naive Bayes classifier. Interestingly, Olah et al. found that cluster 2 is the cluster most enriched for genes that positively correlate with $\beta$-amyloid and tau and is also the cluster with the second strongest enrichment for genes that positively associate with clinical $\mathrm{AD}^{20}$. This is consistent with the finding repeated several 

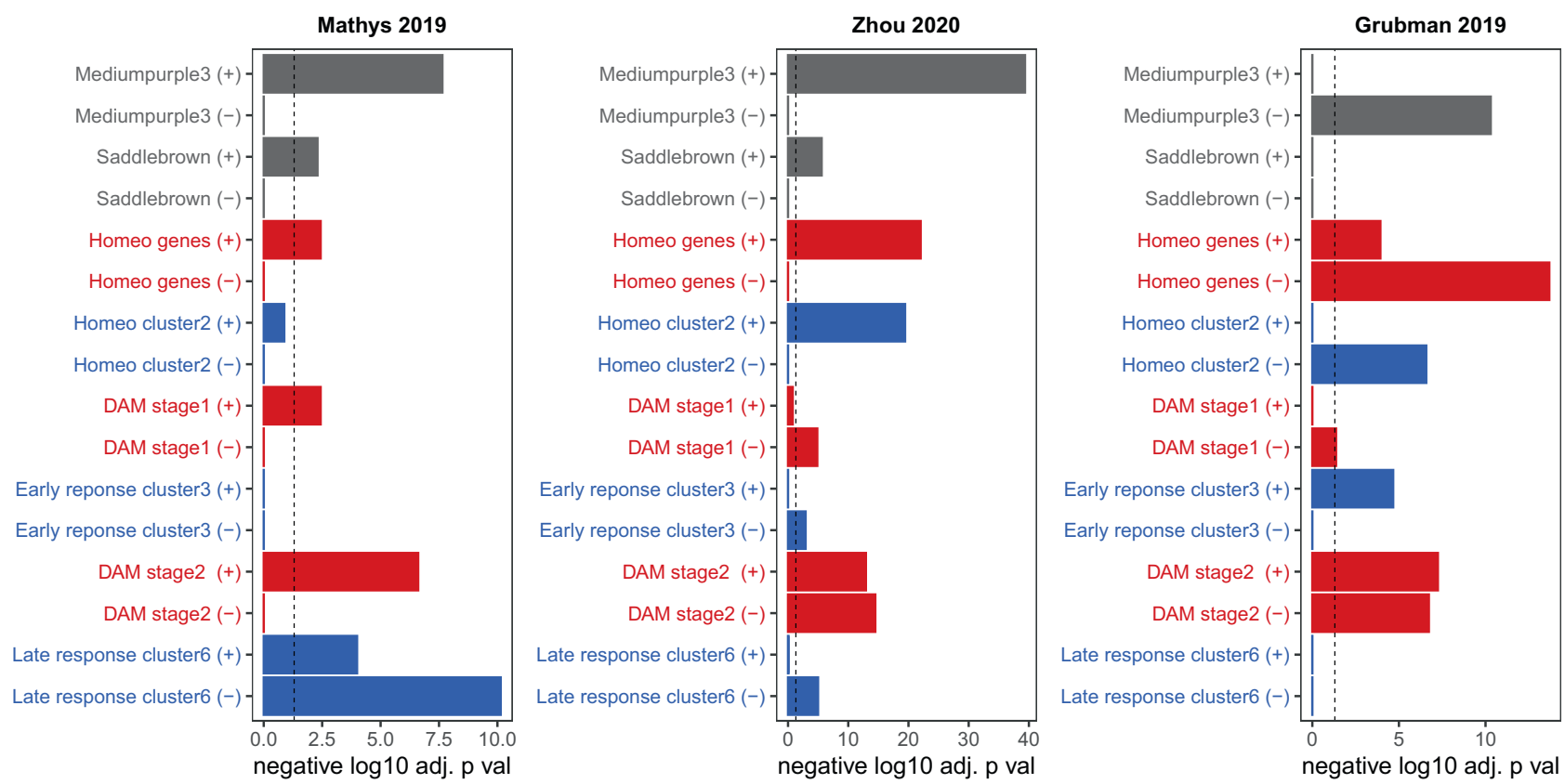

Fig. 6 Human single-nucleus microglial RNA-seq data is enriched for our NPH modules and mouse microglial gene lists. For each single-nucleus RNAseq study, microglial genes that increased $(+)$ or decreased $(-)$ in AD were separately analyzed using two-sided Fisher's exact test for enrichment for gene sets on the $y$-axis; Supplementary Data 7 has all analyses from this figure. The top two modules in gray (mediumpurple3 and saddlebrown) are NPH modules from this paper; mouse gene lists from Keren-Shaul et al..$^{37}$ (red) and Mathys et al. ${ }^{38}$ (blue) are also shown. Mathys et al. ${ }^{21}$ and Zhou et al. ${ }^{19}$ both show enrichment for lists in the microglial gene set that is increasing in AD, although in both datasets there are some DAM or late-stage gene that are decreasing in AD. Similar to the first two studies, Grubman et al. ${ }^{22}$ also shows a mixed response for DAM genes. Unlike the first two studies, Grubman et al. shows significant overlap of homeostatic gene sets with microglial genes that are decreasing in AD. See main text for discussion. Each study is separately Bonferroni adjusted-dotted line in all panels is $p$ value $=0.05$.

times in this manuscript that homeostatic genes positively correlate with $\mathrm{AD}$ traits in autopsy datasets. The cluster most enriched for DAM stage 2 genes is cluster 5 , which they identified as the cluster with the strongest positive association with genes that correlate with clinical $\mathrm{AD}$ and the second strongest positive association with genes that correlate with $\beta$-amyloid (second to cluster 2). Note that, although clusters 2 and 5 are the clusters most enriched for homeostatic and DAM stage 2 genes, respectively, both clusters are also enriched for some mouse gene lists from other categories (Fig. 7). In general, the clusters from Olah et al. are enriched for a broad range of microglial gene lists from the mouse literature (Supplementary Data 7). This suggests that multiple clusters of microglia contribute to the homeostatic or DAM signal in this tissue, and may partially explain why these gene lists co-correlate in late-stage neocortical $\mathrm{AD}$ autopsy tissue (Fig. 5). Alsema et al. also recently profiled single-cell microglia ${ }^{41}$ and found no relevant differences in microglial composition or gene expression between $\mathrm{AD}$ and controls. While this could be for a variety of reasons (as discussed in their paper), we note here that the microglial clusters identified in their manuscript show less overlap with our NPH modules as well as all six mouse microglial gene lists in comparison to the microglial clusters from Olah et al. (see Supplementary Data 7). Thus, the Alsema et al. analysis is less consistent with both our data and the mouse data in comparison to the Olah et al. analysis.

The transcriptomic response of xenograph human microglia in $\mathrm{AD}$ mouse models has recently been studied, and we examined how our modules overlap with and possibly help inform this data. Mancuso et al. recently studied the human microglial response to $\beta$-amyloid injection in an in vivo rodent central nervous system (CNS) environment ${ }^{42}$. This acute treatment led to a microglia population shift away from a homeostatic cluster and toward a cytokine response cluster, and genes that define these clusters overlap significantly with the homeostatic and DAM mouse lists, respectively, as well as with the corresponding modules from this paper (i.e., the homeostatic module overlaps with mediumpurple3 and the cytokine response cluster overlaps with saddlebrown; Supplementary Data 7). Hasselmann et al. recently investigated how human microglia respond in an $\mathrm{AD}$ transgenic mouse model $^{43}$. The authors identified a homeostatic cluster (which did not significantly change in their AD model), as well as a cluster that was enriched for murine DAM genes (which increased in population in $\mathrm{AD}$ transgenic mice). The Hasselmann homeostatic and DAM clusters are strongly enriched for mediumpurple 3 and saddlebrown, respectively, but have some cross-overlap with opposing lists from the mouse literature (Fig. 7). If one assumes that human brain tissue is the actual ground truth, then this suggests that our NPH data may help further clarify which genes identified in common between mouse studies and human xenograph studies are important in the response to early-stage $\mathrm{AD}$ pathology.

NPH modules correlate with microglial histologic features. Finally, in an effort to elucidate the relationship between microglia, $\beta$-amyloid pathology, and our modules, we performed IBA$1 / \beta$-amyloid dual staining in NPH biopsies that we sequenced and correlated our microglial gene expression modules with microglial morphology in the same biopsies. Microglia in biopsies with $\beta$-amyloid plaques tended to be more compact, with an activated, ameboid-like morphology (Figs. $8 \mathrm{a}$ and 6b), and microglial ameboid morphology moderately correlated with plaque area in the same biopsy $(r=0.3 ; p$ value $=0.01 ; n=59$; ameboid morphology was measured using the compactness metric in Cellprofiler-see "Methods" for details). Ameboid 

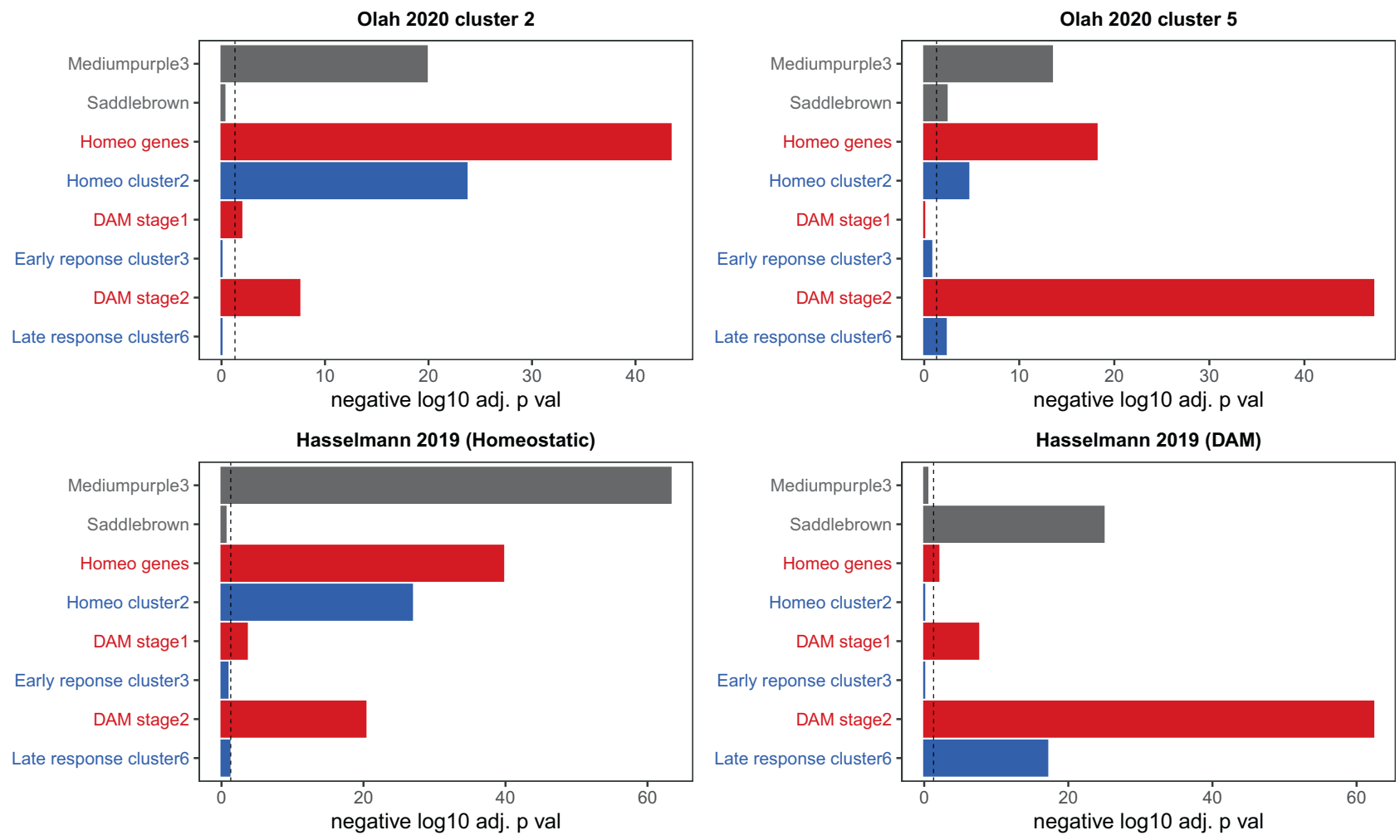

Fig. 7 Human single-cell microglial RNA-seq data is enriched for our NPH modules and mouse microglial gene lists. Clusters 2 and 5 are from single-cell sequencing of microglia from autopsy tissue (Olah et al. ${ }^{20}$ ) and the homeostatic and DAM cluster defining genes from Hasselmann et al. ${ }^{43}$ are from a xenograph mouse model (see text for details). In each panel, the top two modules in gray (mediumpurple3 and saddlebrown) are NPH modules from this paper; mouse gene lists from Keren-Shaul et al. ${ }^{37}$ (red) and Mathys et al. ${ }^{38}$ (blue) are also shown. Cluster 2 from Olah et al. is the cluster from this paper most enriched for homeostatic genes from the mouse literature, and cluster 5 is most enriched for DAM stage 2 genes. Nevertheless, both clusters are also enriched for genes from opposing gene lists (i.e., cluster 2 is enriched for DAM stage 2 genes and cluster 5 is enriched for homeostatic genes), and both are enriched for mediumpurple3. The homeostatic and DAM xenograph clusters are enriched exclusively for mediumpurple3 and saddlebrown, although even here there is some crossover enrichment with mouse lists (i.e., DAM stage 2 gene enrichment with the homeostatic cluster). See main text for discussion and Supplementary Data 7 for all analyses with these datasets, including the analysis displayed in this figure. Each analysis in this figure uses a two-sided Fisher's exact test and is separately Bonferroni adjusted-dotted line in all panels is $p$ value $=0.05$.

morphology also correlated exclusively with the saddlebrown module after Bonferroni adjustment (Fig. 8c; see Supplementary Data 12 for all correlations and $p$ values). In addition to mediumpurple3, we also assessed the correlation of the other three microglial modules from our NPH data shown in Fig. 5. Interestingly, even though several other modules are enriched for disease-associated genes identified in the mouse $\mathrm{AD}$ literature, only saddlebrown significantly correlates with microglial ameboid morphology. The correlation of saddlebrown with microglial morphology is also seen whether or not samples with $\mathrm{AD}$ pathology come from patients who report cognitive symptoms. This suggests that microglial activation and upregulation of genes in the saddlebrown module is an initial response that does not immediately relate to cognitive impairment. Also notable is that mediumpurple 3 is not significant in any comparison, suggesting that loss of homeostatic genes is not related to microglial ameboid morphology in these biopsies.

We also examined the relationship between microglia and plaques in our biopsies, and the relationship with module behavior. Specifically, we looked at the degree of microglial plaque association and normalized by the total plaque area in each analyzed image (Fig. 8d, see "Methods" for details). Interestingly, the mediumpurple 3 module is the only significant module and negatively correlates with microglial plaque association, suggesting that plaque association correlates with loss of homeostatic genes. In contrast, the other microglial modules (including saddlebrown) show no significant correlation with plaque association. When we eliminate samples with $\mathrm{AD}$ pathology from patients either with or without subjective cognitive impairment, we lose significance for mediumpurple3, which suggests that this correlation is weak in this dataset and also that both sample groups are likely contributing to the overall significance of this finding in the full dataset. Noting that saddlebrown correlates with morphology but not with plaque association, we further examined microglial morphology both near and further away from plaques and noted that, in slides with $\beta$-amyloid, both sets of microglia have similar ameboid morphology (Supplementary Fig. 5). Thus, it appears from our data that morphologic changes in microglia occur in the local vicinity of plaques, regardless of whether or not the microglia are physically overlapping the plaque. Although this does not clarify the exact mechanism for how our modules relate to these histologic features, this dissociation between morphology and plaque association is consistent with different modules correlating with these two phenomena. Finally, microglial numbers did not significantly correlate with any of the five microglial modules nor do they correlate with amyloid plaques in our biopsies $(r=-0.12 ; p$ value $=0.35 ; n=59)$, consistent with previous observations that amyloid plaque deposition happens earlier than increases in microglial number ${ }^{44}$, as well as side-by-side comparisons of single-nucleus RNA-seq data showing that the expansion in microglial numbers seen in $\mathrm{AD}$ mouse models is not 
a

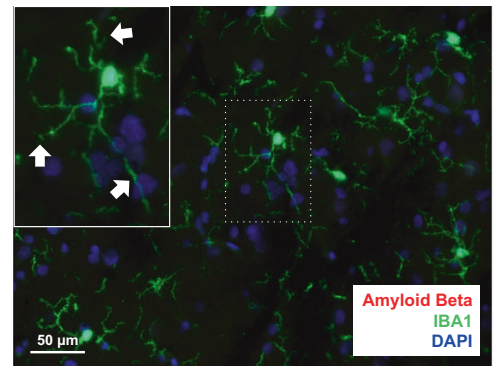

b

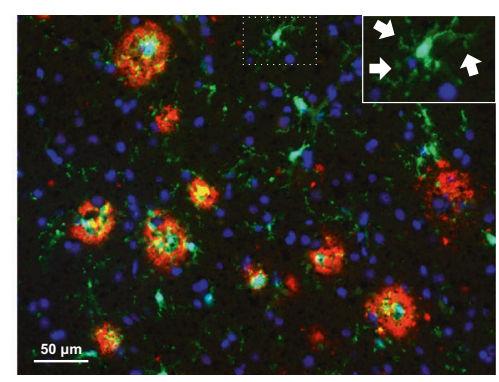

C

WGCNA modules of interest versus Microglial ameboid morphology correlation
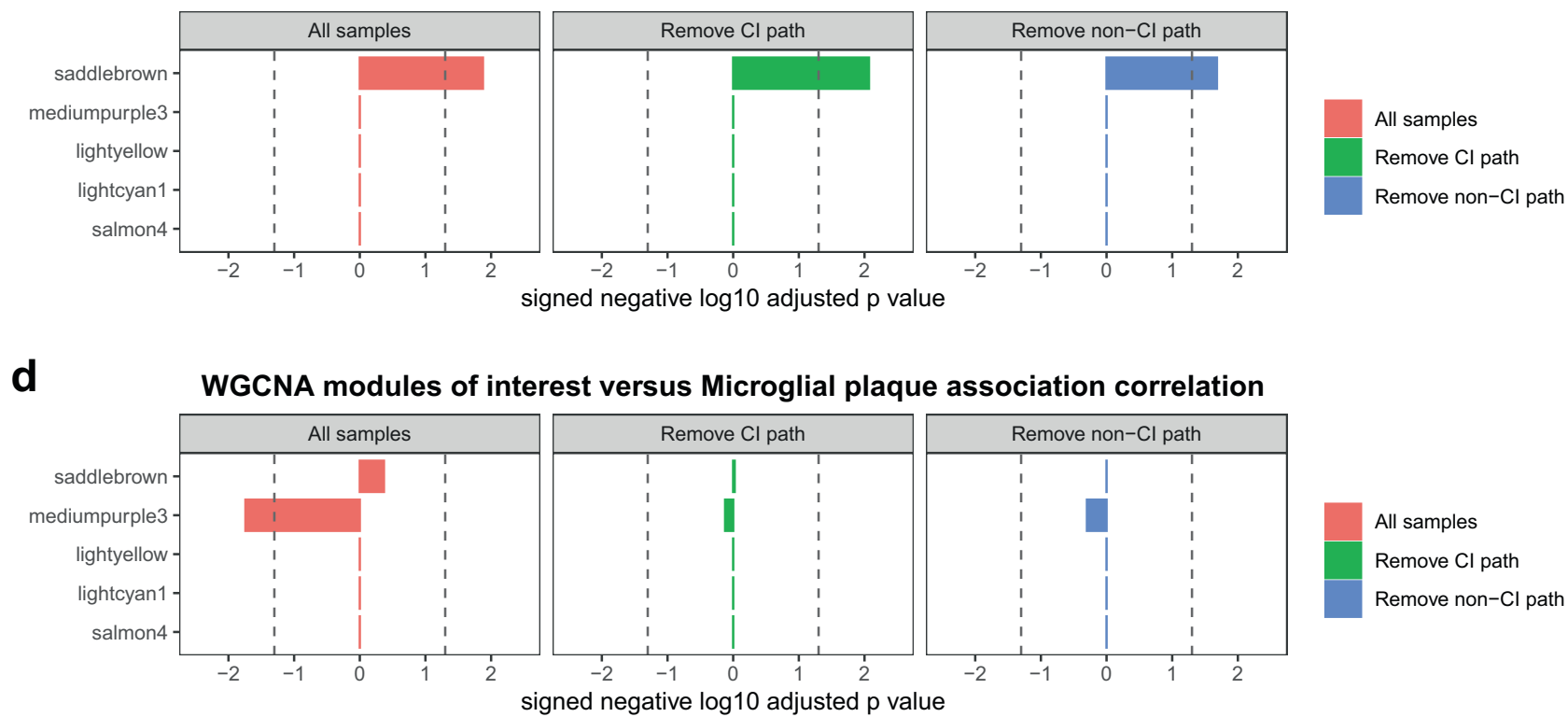

Fig. 8 Our microglial modules correlate with microglial morphology and location. We performed $\beta$-amyloid/IBA-1 dual staining on selected cases from our cohort. a Cases without $\beta$-amyloid showed microglia with longer processes (white arrow; microglial cell in inset is blow-up from area demarcated with dotted line), while $\mathbf{b}$ cases with $\beta$-amyloid pathology tended to show more ameboid, activated microglia. We stained 59 biopsies for $\beta$-amyloid/IBA- 1 and this data is used to calculate correlations in c, $\mathbf{d}$. As noted in the "Results" section, microglial ameboid morphology moderately correlated with plaque area in the same biopsy $(r=0.3$; two-sided $p$ value $=0.01$; ameboid morphology was measured using the compactness metric in Cellprofiler-see "Methods" for details). c, d We assessed the Spearman's correlation with two-sided significance of the top five microglial modules shown in Fig. 5e-i for microglial ameboid morphology (measured using the compactness metric in Cellprofiler-see "Methods" for details) (c) and microglial $\beta$-amyloid plaque association (d). Only saddlebrown significantly correlates with microglial ameboid morphology, and this is seen whether or not samples with AD pathology come from patients who report cognitive symptoms (the green and blue bars represent groups formed using the same methodology as in Fig. 3a, b). In addition, only the mediumpurple3 module significantly (and negatively) correlates with microglial plaque association, suggesting that plaque association correlates with loss of homeostatic genes. When we eliminate samples with AD pathology based on cognitive status, we lose significance for mediumpurple3, which suggests that this correlation is somewhat weak in this dataset, and also that both sample groups are likely contributing to the overall significance of this finding in the full dataset. In c, $n=59$ for all samples (red bars), $n=35$ in the Remove $\mathrm{Cl}$ path group (green bars), and $n=42$ in the Remove non-Cl path group (blue bars); in $\mathbf{d}, n=29$ for all samples, 11 in the Remove $\mathrm{Cl}$ path group (green bars), and $n=18$ in the Remove non-Cl path group (blue bars). The three panels in $\mathbf{c}$ and the three panels in $\mathbf{d}$ are each separately Bonferroni adjusted-dotted line in all panels is $p$ value $=0.05$; see Supplementary Data 12 for all Spearman's correlations, $p$ values, and adjusted $p$ values from this figure.

as prominent in human $\mathrm{AD}$ tissue ${ }^{19}$. One caveat to these observations is that the number of a motile cell type such as microglia counted in a single section of tissue may only loosely correlate with the average number of microglia in the surrounding tissue. Thus, we may not be sufficiently powered to pick up correlations between modules and overall microglial number using this imperfect measure. In summary, the associations of saddlebrown and mediumpurple 3 with microglial morphology and plaque association do not seem to be sensitive to patient cognitive status, in contrast to the association of these modules with accumulating $\mathrm{AD}$ pathology. This is consistent with the view that an initial microglial response to AD pathology is eventually associated with accumulating pathology, non-microglial cell responses, and cognitive dysfunction (see "Discussion").

\section{Discussion}

In this study, we have used cortical biopsies from hydrocephalus patients to examine changes in gene expression that accompany early-stage $\mathrm{AD}$ pathology. Our rationale for examining gene expression and $\mathrm{AD}$ pathology in these biopsies is: (1) This cohort represents a large sample of relatively young patients, many of whom have early-stage AD pathology ${ }^{8,12}$. (2) The tissue in this cohort is also free of gene expression changes that accompany end-of-life hypoxia/apoptosis state, as well as any changes in RNA caused by post-mortem degradation. (3) All cognitive data curated from the patients' charts represent the cognitive state relatively close to the time of tissue acquisition (see "Methods"). The primary findings from this effort are: (1) A limited set of microglial and non-microglial modules correlate primarily with 
AD pathology in patients with subjective cognitive impairment. (2) The microglial modules identified in this analysis correlate in a coherent way with homeostatic and disease-associated gene expression lists from the mouse model literature and with microglial subtypes from the human single-cell literature. (3) In contrast to the existing $\mathrm{AD}$ autopsy literature, these microglial modules replicate both the decrease in homeostatic genes in parallel with an increase in late-stage disease-associated genes seen in the mouse $\mathrm{AD}$ literature. (4) When analyzed in comparison to IBA1- $\beta$-amyloid dual-stained sections, our microglial modules correlate with plaque association and microglial morphology, and this change is not sensitive to cognitive status. Taken together, these data suggest that an initial microglial response is associated with accumulating pathology, nonmicroglial cell responses, and patient-reported cognitive status.

As noted in the "Results" section, we have identified a gene expression module enriched for murine disease-associated microglial genes that positively correlates with $\mathrm{AD}$ pathology (saddlebrown) and a module enriched for murine homeostatic microglial genes that negatively correlates with $\mathrm{AD}$ pathology (mediumpurple3), and in aggregate, this is more consistent with the existing mouse literature than publicly available $\mathrm{AD}$ autopsy datasets ${ }^{1-4,19,21,22}$. Although it is not entirely clear why our data more closely aligns with the mouse $\mathrm{AD}$ literature than existing autopsy datasets, there are several important differences between our data and human $\mathrm{AD}$ autopsy data that may influence the differences we are seeing. One obvious difference is that our tissue was obtained in a different way from AD autopsy tissue. Specifically, our tissue was removed from living cortex while the patient is under anesthesia, as opposed to being acquired after patient death. In this regard, the lack of any post-mortem artifact in our data makes it more similar to tissue obtained from laboratory mice, and one could speculate that post-mortem factors may partially obscure the $\mathrm{AD}$-associated microglial response. Although this presents an intriguing possibility for how our data may be more similar to the mouse literature, an alternative (and not mutually exclusive) explanation is that hypoxia and/or sepsis at the end of life could be affecting the CNS inflammatory response in a way that obscures or further obscures the ADassociated microglial transcriptomic response. With regard to the downregulation of homeostatic genes, we did identify one autopsy dataset (in entorhinal cortex) that shows a subset of homeostatic genes that decreases in $\mathrm{AD}$, which somewhat mitigates a purely artifactual explanation for why the majority of the autopsy datasets show no change or an increase in homeostatic genes in AD. Note, however, that the entorhinal cortex study (Grubman et al. ${ }^{22}$ ), similarly to the neocortical studies of Zhou et al. ${ }^{19}$ and Mathys et al. ${ }^{21}$, also shows a mix of increasing and decreasing DAM genes in $\mathrm{AD}$, with an almost even split for the DAM stage 2 genes from Keren-Shaul et al. ${ }^{37}$. As noted earlier, one possible explanation for this comes from a recent study by Thrupp et al. ${ }^{40}$ which suggests that DAM genes in particular may be missing from single-nucleus RNA-seq, which only captures nuclear RNA. In fact, this study directly compared the genes depleted in singlenucleus RNA-seq vs. single-cell RNA-seq to the data from Mathys et al. and showed that most of the DAM genes highlighted in the Mathys et al. paper were the subset of DAM genes that had relatively higher expression in the nucleus. Note that Thrupp et al. did not include disease tissue in their analysis, and it is not clear whether DAM genes are more or less expressed in the nucleus in $\mathrm{AD}$ in comparison to their data. How much the Thrupp et al. finding may affect the analysis of nuclear RNA-seq studies presented here is unclear, but this could be an important factor explaining discrepancies between the singlenucleus RNA-seq data and the bulk RNA-seq data presented in this manuscript.
Of course, we should also note that our biopsies represent an earlier stage of pathology than most autopsy cohorts. Indeed, one major caveat to this study is that these patients do not have $\mathrm{AD}$ per se, but rather $\mathrm{AD}$ pathology. As mentioned in the "Introduction" section, NPH patients with AD pathology are less responsive to shunting 8,13 and are at an increased risk of developing $\mathrm{AD}^{6}$, which supports studying this cohort as a group that may represent a pre-AD population. In addition, $\mathrm{AD}$ is clinically defined as $\mathrm{AD}$ pathology in the setting of cognitive impairment on a neuropsychology exam ${ }^{45}$, and our changes in gene expression are most robust in patients who report subjective cognitive impairment, which suggests that what we are seeing in these biopsies is indeed relevant for the earliest stages of AD. However, our metric for measuring cognitive decline is based on subjective reporting by the patient during a contemporaneous clinical exam. We used this metric because standardized neuropsychology testing was not carried out on all patients, while documentation of the patient-reported subjective cognitive impairment was found in a large majority (93) of the patient files for the biopsies sequenced in this study. Although subjective cognitive decline correlates with a patient's psychometric assessment ${ }^{46}$, it is not as precise as formal neuropsychology testing. Another major caveat is that $\mathrm{AD}$ pathology is common in elderly subjects without cognitive impairment, many of whom die before ever developing $\mathrm{AD}$ dementia ${ }^{16-18}$, and the biological meaning of $\mathrm{AD}$ pathology in subjects without cognitive impairment is still an area of investigation ${ }^{47-49}$. This is particularly relevant because the metric for measuring cognition in this study is not precise, so we cannot definitively say which patients in our study are pre-AD. One possible interpretation of our data is that $\mathrm{AD}$ pathology is associated with a more robust biological effect in patients with subjective cognitive decline because these patients are moving into the earliest stage of $\mathrm{AD}$, and the relative lack of significant genes in our analysis overall is a reflection of this early point in the disease trajectory. However, a more careful study of this patient population with formal neuropsychology testing and longitudinal follow-up would be necessary to definitively say which patients will progress to clinical AD.

Note that other studies have included early AD groups, although these groups often have more pathology on average than our cohort. For example, Mathys et al. ${ }^{21}$ has an early AD cohort in their study. However, 8 of the 15 subjects in this group are Braak stage 5, which would indicate significant cortical tau pathology in half of their early AD subjects (although this is less pathology than in their late-stage subjects). As noted in Fig. 5, microglial gene groups from the mouse literature co-correlate in the ROSMAP modules. This mixing of microglial responses in the ROSMAP microglial modules may partially explain the relative lack of correlation of these modules with $\mathrm{AD}$ pathology in Mostafavi et al. ${ }^{1}$. However, as noted in their paper, their module 116 did correlate with AD pathology when analyzed in the data of Zhang et $\mathrm{al}^{3}$. Although untangling the reasons for all of these discrepancies is outside the scope of this study, we note here that the general phenomenon of co-correlation raises the possibility that a general microgliosis and increased microglia infiltration late in $\mathrm{AD}$ may cause the apparent reversal of the decline in homeostatic genes. However, note that an upregulation of homeostatic genes in human AD autopsy tissue is seen in several single-nucleus and single-cell RNA-seq studies analyzed in this paper. This eliminates the possibility that increased homeostatic gene expression in later-stage $\mathrm{AD}$ is entirely due to changes in cellular composition and points to an actual change in microglial gene transcription. Confounding all of these analyses is the additional issue that all of these autopsy cohorts are also older than ours. For example, the mean age of the ROSMAP dataset is 88.7 years $^{1}$, the mean age of the MSSM dataset is 84.7 years $^{4}$, and 
the Mathys et al. cohort has an average age of 86.7 years for the $\mathrm{AD}$ group and 87.1 years for the non-pathology group ${ }^{21}$.

Nevertheless, our data suggest that the downregulation of homeostatic genes in conjunction with an increase in late-stage/ disease-associated genes is occurring at the earliest stages of $\mathrm{AD}$ pathology in neocortex. The implication of this work is that the $\mathrm{AD}$ mouse literature may be modeling the earliest stages of the microglial response to $\mathrm{AD}$ pathology in a more faithful way than previously recognized. The Grubman et al. study is the most similar to our cortical NPH biopsies. Although this is only one study, one could speculate that entorhinal cortex and neocortex may have an initial common microglial response to $\mathrm{AD}$ pathology that diverges in neocortex as pathology accumulates. Future work will be needed to better determine the similarities and differences between the microglial response in entorhinal cortex and neocortex.

It should be noted that three of the top five microglial modules in our WGCNA analysis by enrichment (lightyellow, lightcyan1, and salmon4) do not correlate with any of the pathologic metrics in this paper, despite being significantly enriched for several groups of disease-associated genes from the mouse literature (Fig. 5). This highlights an obvious point that the microglial response in mice is not exactly the same as in humans. However, it also suggests that the data in this paper can serve as a conceptual bridge between some of the early responses seen in the mouse literature and the human $\mathrm{AD}$ literature. While there will always be interspecies differences in these comparisons, the closer similarity of our data to the mouse literature suggests that our data may help clarify which aspects of mouse biology may be accurately modeling the early microglial response in humans.

It should also be noted that while the Keren-Shaul et al. study used an APP-based model $(5 \times \mathrm{FAD})^{37,50}$, Mathys et al. used the CK-p25 model, which shows elevated $\beta$-amyloid as well as neurofibrillary tangles $38,51,52$. Similarities and differences in the immune response to different neurodegenerative disease pathologies is an area of active research, and future work will be needed to determine which findings from this paper are specific for different $\mathrm{AD}$ pathologies or generalizable to other neurodegenerative diseases. Indeed, although we have highlighted the microglial response in this paper, there is no a priori reason to assume that the microglial response in our data is pathogenic or even specific for AD. This highlights a related point that our work also identifies non-microglial genes as being important in the earliest stages of AD pathology. Although significant genes at the individual level are mostly microglial (Fig. 2), it should be noted that several of the individual genes that reach significance in our analysis are astrocytic. For example, GFAP is a reactive astrocyte marker, and CD44, SERPINA3, and C4B have all been associated with disease-associated astrocytes ${ }^{19,27,28}$. While microglia are the predominant cell type represented in our transcriptomic data, an astrocytic response is also clearly present at this early stage of $\mathrm{AD}$ pathology even at the single-gene level. This observation is further supported by our WGCNA analysis, which identifies an astrocytic (orange) and neuronal (darkgrey) module in addition to our two microglial modules. One might therefore look at the orange and darkgrey modules for astrocytic and neuronal genes that are involved in early cognitive decline in the setting of a prolonged microglial response.

Human tissue samples are often acquired for clinical and circumstantial reasons beyond the control of the researcher, and the tissue used in this study also has important limitations for similar reasons. Most obviously, all of the patients in this study have the co-morbitidy of hydrocephalus. Hydrocephalus biopsies are collectable from alive individuals and therefore do not present some of the limitations of analyzing post-mortem brain tissue. However, the presence of hydrocephalus can also be a confounding factor and it is not easy to disentangle what effect this might have on gene correlations with AD pathology. Never the less, there are several reasons to hypothesize that the effect of hydrocephalus may be minimal when comparing the data in this study to other gene expression studies on human $\mathrm{AD}$ autopsy brain tissue. First, $\mathrm{AD}$ is rarely pure in a pathologic sense. In recent years, there has been a growing appreciation that dementia in the elderly is often due to several co-morbid conditions in any individual, as vascular disease, Lewy body dementia, and TDP-43 pathology are frequently seen in AD autopsy cohorts ${ }^{53-55}$. Thus, pure $\mathrm{AD}$ is actually less common among patients with dementia than mixed pathology. Previously reported gene correlations with $\mathrm{AD}$ pathology were presumably found in spite of these common confounders, and there is no a priori reason to expect hydrocephalus to uniquely affect these correlations more than other common confounders. In addition, it should again be noted that, although we have found far fewer changes in gene expression in comparison to previous studies of $\mathrm{AD}$ brain tissue, we have also found other changes (i.e., a loss of homeostatic genes) that are not found in $\mathrm{AD}$ autopsy cohorts but are found to some extent in $\mathrm{AD}$ animal models. Although one could theorize that findings in this study are discordant with the AD autopsy literature due to hydrocephalus, the alignment of these findings with the $\mathrm{AD}$ animal literature strongly suggests that we are observing the same phenotypic change that has been documented multiple times in AD animal models.

In conclusion, this study identifies a restricted set of genes that correlate with early $\mathrm{AD}$ cortical pathology and subjective cognitive impairment and points to future directions for research into how microglia may mediate early cognitive decline. In addition, this work identifies NPH patients with AD pathology as a possible pre-AD population that may benefit from early intervention in AD clinical trials. Finally, this study suggests that this patient population may be particularly interesting to study prospectively, and future studies will seek to link these gene expression modules with subsequent cognitive decline or cognitive resilience.

\section{Methods}

NPH biopsy sample collection and histopathology studies. This study was reviewed and approved by the Columbia University Review Board (study plan IRBAAAT7985), and all relevant ethical regulations have been followed. This study is a retrospective study that uses residual tissue samples not required for clinical diagnosis and associated clinical and demographic data. At the time of surgery, a portion of tissue was frozen and deposited in the Bartoli Brain Tumour Bank at Columbia University. Patients undergoing surgery signed an umbrella consent form, providing consent to use the remaining biopsies and body fluids for research. A portion of tissue was submitted for clinical analysis. After completion of the clinical tests, any residual tissue can be released by the tissue bank for research purposes. Tissue and clinical data used in this study were provided de-identified by the tissue bank.

NPH was originally defined by ventricular dilation with normal CSF pressure, with a classical clinical triad of imbalance/ataxia; cognitive impairment, particularly short-term memory decline; and urinary incontinence 56,57 . The usefulness of the concept of NPH has been more recently challenged ${ }^{58,59}$ due to the fact that the symptoms in this clinical triad are common in the elderly population at large ${ }^{59}$; that (conversely) all three symptoms may not actually occur in all patients diagnosed with $\mathrm{NPH}^{59,60}$; and that the CSF pressure in NPH patients can actually be variably elevated ${ }^{10,61}$, suggesting a chronic deficit in CSF resorption. Not surprisingly, NPH may sometimes arise secondarily to known medical conditions that affect CSF resorption, leading to the concept of secondary NPH, to be distinguished from iNPH $9,10,58,59$. Patients suspected of having NPH can be clinically stratified into probable NPH vs. possible NPH, depending on how well the patient's symptoms match the appropriate clinical picture and whether any comorbidities may also be accounting for the patient's clinical and imaging findings ${ }^{60}$. We performed RNA-seq on 106 biopsies from NPH patients, 90 of which fell within the definition of probable or possible iNPH, according to the criteria of Relkin et al. ${ }^{60}$ (16 biopsies came from patients with a known benign lesion near the cerebral aqueduct that may have contributed to the patient's chronic hydrocephalus, which would qualify as secondary NPH). Our cohort of 106 samples has an average age of 74.9 years (standard deviation 8), with 42 females and 64 males. In all cases, patients were shunted for chronic hydrocephalus by the same surgical team, and biopsies were taken from either frontal cortex 
(middle frontal gyrus at coronal suture) or parietal cortex ( $\sim 4 \mathrm{~cm}$ off midline in parietal lobe, just above parieto-occipital junction). The decision to shunt/biopsy in frontal or parietal cortex was made by the surgeon based on cosmetic considerations; in total, approximately $2 / 3$ of our cohort have frontal biopsies and $1 / 3$ have parietal biopsies. Cortical biopsies were divided in the operating room immediately after removal, and half of each biopsy was frozen in liquid nitrogen while the other half was formalin fixed and paraffin embedded for subsequent pathology diagnosis (Fig. 1)

In addition to a hematoxylin and eosin stain, immunohistochemistry was performed with antibodies against tau (AT8 at 1:200 dilution; Thermo Fisher; Catalog \# MN1020), $\beta$-amyloid (6E10 at 1:200 dilution; BioLegend; Catalog \# 803003), $\alpha$-synuclein (KM51 at 1:40 dilution; Leica; Catalog \# NCL-L-ASYN), and TDP-43 (C-terminal rabbit polyclonal at 1:500 dilution; Proteintech; Catalog \# 12892-1-AP). All slides were counterstained with hematoxylin. Immunostaining was performed in the Ventana automated slide stainer without manual antigen retrieval and was detected using the Ventana ultraView Universal DAB Detection Kit (Tucson, AZ) as recommended by the manufacturer. Patients in this study had variable amounts of $\beta$-amyloid and tau pathology and no $\alpha$-synuclein or TDP-43 pathology or any other visible diagnostic abnormality on hematoxylin and eosin staining. $\beta$-Amyloid plaques were counted per square $\mathrm{mm}$; in slides with enough tissue, three fields were averaged together, whereas in slides with less tissue, the largest number of possible fields were counted. For tau quantification, we devised a rating scale to grade the minimal degree of tau pathology seen in NPH biopsies (see Supplementary Fig. 1). Grade 0 was given to biopsies with no tau pathology. Grade 1 was given to biopsies that have any tau pathology at all, usually one or more dystrophic neurites, but do not make criteria for Grade 2. Grade 2 was given to biopsies that have at least one tau-positive neuron or neuritic plaque, but do not make criteria for Grade 3. Grade 3 was reserved for biopsies with tau pathology evenly distributed throughout the biopsy.

Note that a Braak stage cannot be assigned to these biopsies, as Braak staging is a global assessment of tau pathology that takes into account the presence and density of tau in multiple regions ${ }^{62}$. Although tau begins to spread broadly into neocortex at Braak stage $4, \mathrm{AD}$ tau pathology shows natural variability from case to case, and occasional focal staining of higher Braak stage areas can be observed at earlier Braak stages ${ }^{62}$. Thus, in the absence of additional anatomical data Braak staging is not possible. Moreover, it should be emphasized that the primary purpose of the tau grading system devised in this manuscript is to measure the local density of pathology and compare it to local changes in gene expression. All of these considerations apply to Thal staging of $\beta$-amyloid as well (i.e., Thal stage requires a global assessment of $\beta$-amyloid burden) ${ }^{63}$. In this case, note that $\beta$ amyloid appears initially in neocortex (Thal stage 1), so all of our biopsies with $\beta$ amyloid would qualify as Thal stage 1 , and we cannot stage higher using only neocortical tissue.

Additional patient data (sex, age, NPH diagnosis, and subjective cognitive status on intake exam) were gathered from the medical record. Cognitive exam data were gathered from patient medical exams as close to the time of biopsy as possible. If possible, an exam note was located where the patient was asked whether they had experienced subjective cognitive impairment. Using this simple metric (yes vs. no), we were able to assign 93 of our sequenced biopsies into yes or no, with 59 replying yes and 34 replying no (the remaining 13 biopsies came from patients with no clear answer from the medical record). The average time between exam and biopsy among all 93 samples was 120 days. Patients who reported subjective cognitive impairment had non-significantly higher $\beta$-amyloid and tau load than patients who reported no cognitive impairment ( $p$ value for $\beta$-amyloid $=0.21 ; p$ value for tau $=0.66$ by Mann-Whitney $U$ test). Interestingly, all of the tau grade 3 biopsies with cognitive information have a history of subjective cognitive complaint. However, these biopsies are so few in number (7) that they do not significantly affect the overall analysis (see Supplementary Table 1 for distribution of samples by cognitive status).

NPH sample RNA sequencing and data preprocessing. RNA was extracted from biopsy samples using the miRNeasy Mini Kit (QIAGEN; Cat No./ID: 217004), which purifies total RNA including miRNA (note that our library prep protocol selects poly(A) coding mRNA). RNA integrity was measured on an Agilent Bioanalyzer, and samples with RNA integrity number (RIN) values $\geq 6$ were selected for sequencing. RNAs were prepared for sequencing using the Illumina TruSeq mRNA Library Prep Kit, and samples were sequenced with Illumina HiSeq 2000, 2500, and 4000 (potential batch effect attributable to different sequencers was regressed out along with other confounding variables using surrogate variable analysis; see below), and all samples underwent single-end sequencing to $30 \mathrm{M}$ read depth. The quality of all fastq files was confirmed with FastQC v 0.11.8 ${ }^{64}$. Fastq files that passed quality check were further mapped to Genome Reference Consortium Human Build 37 (GRCh37) reference genome with STAR ${ }^{65}$. Output BAM files from STAR were further processed with featureCounts ${ }^{66}$ to obtain raw counts for each gene of all the sequenced samples.

Genes with $<5$ counts in at least $90 \%$ of all samples were first filtered out from the raw count matrix, followed by variance stabilizing transformation (VST) on filtered counts utilizing the varianceStabilizingTransformation function from DESeq2 R package ${ }^{67}$. VST is a widely applied strategy that transforms data from a distribution of fluctuating variance into a new distribution with nearly constant variance in order to facilitate downstream analysis ${ }^{68}$. After VST, surrogate variable analysis (SVA ${ }^{23}$ was used to identify variation in gene expression not attributable to $\beta$-amyloid load or tau load. Specifically, a full model (with primary variables to be kept) and a null model (with intercept only) were built using the model.matrix() function, and then sva() function from sva $r$ package was applied to determine surrogate variables (SVs) from the VST-processed gene expression matrix, in which sva function parameter dat was assigned with the gene expression matrix, mod as the full model, mod0 as the null model and method = irw. The identified SVs, which represent known and unknown confounding variables in our dataset, were later regressed out with removeBatchEffect function from limma R package ${ }^{69}$. The filtered, VST-processed, and surrogate variable regressed count matrix was used for all downstream analyses in this manuscript. As an additional exercise, we also attempted to regress out confounding variables individually. To do this, the NPH expression matrix was first quantile-normalized, followed by $\log 2$ transformation, and then the batch effect was removed through the ComBat $\mathrm{R}$ function from the sva package, and finally age, gender, and RIN were regressed out using the removeBatchEffect $\mathrm{R}$ function from the limma package. This method did not yield any significant genes at an FDR threshold of 0.1 , confirming the relatively minimal transcriptomic changes in this tissue cohort. This also highlights the need to regress out both known and hidden confounders in our dataset in order to detect the relatively minimal changes in gene expression that we do find. Finally, we also performed differential gene expression analysis on the NPH count data as an additional exercise and used DESeq2 ${ }^{67}$ to do the following comparisons: (1) No AD pathology (no $\beta$-amyloid or tau, $n=32$ ) vs. any AD pathology (either $\beta$-amyloid and/or tau, $n=74)$, (2) No $\beta$-amyloid pathology $(n=49)$ vs. any $\beta$-amyloid pathology $(n=57)$, and (3) No tau $(n=42)$ vs. any tau $(n=64)$. These analysis yielded 2, 19, and 4 genes passing FDR 0.1, respectively (Supplementary Data 1). These results further confirm the overall consistency of gene expression signatures in these biopsies and supports our view that this tissue is from patients with the earliest stages of $\mathrm{AD}$ pathology, before more extensive pathophysiologic changes have occurred.

For our analysis below, we combine RNA-seq data from all biopsies to achieve higher statistical power; this analysis is effectively investigating changes in gene expression shared by two areas of neocortex related to early $\mathrm{AD}$ pathology. This power is necessary to detect the relatively subtle changes in gene expression accompanying these early changes in AD pathology (see below). Analyzing frontal and parietal areas separately showed that both analyses trended in a way similar to the combined dataset (see Supplementary Data 1).

RNA-seq data analysis and module characterization. For single-gene analysis, we calculated the Spearman's correlation between $\beta$-amyloid and tau burden and individual genes using the cor.test function in R. The $p$ values were further Benjamini-Hochberg (BH) adjusted across all the genes in the dataset using the p.adjust function in R. To generate gene expression modules, we utilized WGCNA to identify gene co-expression modules ${ }^{29}$, on the default (unsigned) setting, with softPower $=7$ and minModuleSize $=20$. The eigengene for each WGCNA module was correlated with $\beta$-amyloid and tau burden and eight cell-type-specific signatures from the human single-nucleus RNA-seq literature ${ }^{21}$ (Inhibitory neurons, Excitatory neurons, Oligodendrocyte precursor cells, Oligodendrocytes, Astrocytes, Microglia, Endothelial cells, and Pericytes). Note that for all correlations of WGCNA eigengenes with other gene lists (including the cell-type-specific gene lists above and mouse and human microglial subtypes below), the WGCNA eigengene is correlated with the mean gene expression vector of these various lists. While the eigengene is the preferred way of capturing the variance of highly correlated WGCNA gene modules ${ }^{29}$, there is no a priori reason to assume that the eigengene will capture the majority of the variation in gene lists that are not formed though measuring intercorrelations and thus may not be as highly intercorrelated as WGCNA modules. Thus, for cell-type and microglial gene lists we use the mean gene expression vector as a more holistic measure of variation of these gene lists.

To identify cell class-specific genes from single-nucleus RNA-seq data from the Mathys et al. study ${ }^{21}$, we first used edgeR to calculate differential expression among all pairs of broad cell classes annotated in the study; each individual nucleus was treated as a sample in edgeR, and un-normalized count values were used. For each of these pairs, we then selected cell class-specific genes as those having at least 1.5 positive fold change (FC) between the class of interest and the mean transcripts per million (TPM) value of all other classes in a pairwise fashion, with the added condition that a given candidate gene mean TPM value should be larger or at least equal to 20 . The selected genes were further ranked in descending order based on FCs, from which the mean expression of the top 500 genes were used for correlation analysis.

In addition, FET was performed to check the enrichment of cell type for the group of individual genes that passed FDR threshold or specific modules of interest. For enrichment analysis, all cell-type-specific genes that passed the filtering criteria for FPKM (fragments per kilobase per million) and FC were selected for the test. The FET $p$ values were Bonferroni corrected across all cell types for each comparison. The ontology analysis was performed with all the genes in a given module using overrepresentation analysis under the gene set analysis tab from ConsensusPathDB web tool $^{70}$. All the level 2 and 3 ontology groups, including all three categories (molecular function (m), biological process (b), and cellular component (c)), with at least two shared genes with the test module were selected and enrichment $p$ values were calculated through FET. The $q$-values were FDR adjusted across all the selected 
ontology terms within each category ( $m, b$, or $c)$. All selected ontology groups were further ranked in ascending order based on $q$-values, and the top 10 most significantly enriched pathways are listed in Fig. 4.

Note that, for this study, we have employed an FDR threshold of 0.1 when screening large numbers of hypotheses to identify genes or modules of interest (i.e., genes or WGCNA modules that correlate with AD pathology or ontology groups that are enriched for module genes). Throughout the rest of the manuscript, tests of hypotheses about these genes/modules have employed a Bonferroni correction with a $p$ value threshold of 0.05 .

Comparison of NPH modules with AD autopsy datasets. We compared NPH datasets with two publicly available $\mathrm{AD}$ autopsy datasets:

1) The ROSMAP Study (Synapse ID: syn3219045)

RNA-seq raw counts in total 596 human subjects were obtained from paired-end fastq files using STAR + featureCounts pipeline for pair-ended reads. The raw counts matrix was filtered and VST transformed as described above. Finally, variation in gene expression not attributable to Braak stage, CERAD score, and MMSE was regressed out using SVA, similar to our processing pipeline for NPH data.

2) The Mount Sinai Brain Bank Study (Synapse ID: syn3159438)

We downloaded 183 bulk RNA-seq raw count tables from human brain samples from Brodmann area 10. Samples were preprocessed the same way as our NPH samples and the ROSMAP data, and SVA was used to regress out variability not attributable to Braak stage, CERAD score, and CDR score.

Effects of cognitive status on NPH AD traits. In Supplementary Fig. 2, patients who report subjective cognitive impairment are compared to patients who report no cognitive impairment. The cognitive information of each subject was obtained as described above. Mann-Whitney $U$ test was first performed to examine the difference in average $\beta$-amyloid and tau load between subjects who report cognitive impairment and subjects who report no cognitive impairment. To further explore the distribution of these variables, we first performed FET on whether tau and $\beta$ amyloid significantly co-occur in biopsies from patients with reported cognitive impairment vs. biopsies from patients who report no cognitive impairment. We next performed a Mantel-Haenszel test of whether the odds ratios between the two groups were different (Supplementary Fig. 2). In Fig. 3a, b, we asked whether samples with $\mathrm{AD}$ pathology from either group were significantly driving the correlation between the modules and $\mathrm{AD}$ pathology. To do this, we first removed all samples with AD pathology from our cohort where patients reported cognitive impairment (our Remove CI path group, $n=66$ ). Next, we removed all samples with $\mathrm{AD}$ pathology where patients who reported no cognitive impairment (our Remove non-CI path group, $n=80$ ). Note that, in both groups, all biopsies without $\mathrm{AD}$ pathology were included. Finally, we ran 1000 iterations where half of the samples with $\mathrm{AD}$ pathology from the Remove non-CI path group (20 out of 40 ) were randomly selected to be replaced by another randomly selected 20 samples with pathology from the Remove CI path group to form an artificial Remove nonCI path group (i.e., pathology samples with subjective cognitive impairment are being randomly replaced with pathology samples without documented cognitive impairment). Mann-Whitney test of $\beta$-amyloid or tau between the real and artificial groups and correlations of module eigengenes with $\beta$-amyloid or tau in both groups were performed for each iteration and $p$ values from these tests were recorded. The correlation $p$ values were further BH adjusted across all WGCNA modules. At the end of all iterations, the number of times out of 1000 iterations (1) Mann-Whitney test $p$ value was $<0.05$ for $\beta$-amyloid or tau and (2) the BH adjusted correlation $p$ values that passed significance threshold (0.1) for any of the four modules in Fig. 3 was reported. As noted in Supplementary Data 4, this did not statistically change the overall burden of pathology in any of the simulations. In contrast, all four modules of interest fail to pass 0.1 FDR significance in their correlation with $\beta$-amyloid and tau for the majority of the simulations.

Microglia activation stage- and subtype-specific gene identification. Microglia activation stage-specific genes from mouse studies were obtained from two separate datasets. We used three sets of genes reported in Keren-Shaul et al. ${ }^{37}$ (Homeostatic genes, DAM stage 1, and DAM stage 2) and three sets of genes reported in Mathys et al. ${ }^{38}$ (Homeostatic cluster 2, Early response cluster 3 (the primary early response cluster), and Late response cluster 6). We defined cell-type-specific genes using comparisons done in these manuscripts. Specifically, homeostatic genes from Keren-Shaul et al. were defined as differentially expressed (DE) genes with a minimum twofold increase in expression compared to Tg $5 \times \mathrm{FAD}$ samples and FDR $<0.1$. The DAM stage 1 and DAM stage 2 genes were filtered through similar criteria except that the DE genes were calculated based on the comparison between these two DAM stages. For gene lists from Mathys et al. (i.e., clusters 2, 3, and 6), cluster-specific genes were defined as genes upregulated in a given cluster in comparison to either of the other two clusters and not downregulated in either of these two comparisons. The significance of these comparisons was defined as absolute value of the corrected $z$ score $>1.25$, which is equivalent to an FDR corrected $p$ value $>0.1$. Mouse gene symbols were converted to human gene symbols with $\mathrm{R}$ biomaRt package for comparison with the data in this paper.
Immunofluorescence staining and imaging. Immunofluorescence was performed on cortical biopsies for $\beta$-amyloid and IBA-1 to visualize microglial morphology and microglial plaque association. Fixed, paraffin-embedded tissue was sectioned at $7 \mu \mathrm{m}$, and slides were submerged in two washes of Histo-clear II (National Diagnostics HS-202) for $10 \mathrm{~min}$ each, before being washed in the following series for 1 min each: $100 \%$ ethanol, $100 \%$ ethanol, $70 \%$ ethanol, and MQ water. They were then washed 3 times in Tris-buffered saline (TBS) for 5 min each. Antigen retrieval was performed by incubating the slides in citrate buffer for $25 \mathrm{~min}$ in a microwave set to 400 Watts. The slides in citrate buffer were rested at room temperature for $30 \mathrm{~min}$ before a 5-min wash with $\mathrm{MQ}$ water, two 5-min permeabilization washes in TBS with $0.25 \%$ Triton X-100 (ACROS Organics), and a 5 -min wash in TBS. The sections were blocked with $10 \%$ donkey serum (Abcam ab7475) and 1\% bovine serum albumin (BSA; Sigma-Aldrich A7284) in TBS for $1 \mathrm{~h}$ at room temperature. A primary antibody solution was created in 1\% BSA in TBS, with the addition of mouse polyclonal anti- $\beta$-amyloid (1:200; Cell Signaling Catalog \# 15126, lot 1) and rabbit polyclonal anti-IBA1 (1:500; Wako Catalog \# 019-19741, lot ptr2404). The sections were stained overnight in the primary antibody solution at $4{ }^{\circ} \mathrm{C}$, after which they underwent three 5 -min washes in TBS. A secondary antibody solution was created in 1\% BSA in TBS, with the addition of Alexa Fluor 555-conjugated Donkey Anti-Mouse (1:1000; Invitrogen A-31570, lot 1850121) and Alexa Fluor 488-conjugated Donkey Anti-Rabbit (1:1000; Invitrogen A-21206, lot 1981155). The sections were incubated for $1 \mathrm{~h}$ at room temperature in the secondary antibody solution, after which they underwent three 5 -min washes in TBS. To block autofluorescence, Sudan Black B (EMS 21610) solution at $0.1 \% \mathrm{~m} / \mathrm{v}$ in $30 \% \mathrm{MQ}$ water and $70 \%$ ethanol was placed on the sections for $20 \mathrm{~min}$, after which they underwent three 5-min washes in TBS Nuclei were labeled with 4,6-diamidino-2-phenylindole (1:4000 from stock; Invitrogen D1306) in TBS for $5 \mathrm{~min}$, before three 1-min washes in TBS. Slides were mounted with Vectashield (Vector H-1000).

Cellprofiler was used to perform image analysis on the immunohistochemistrystained slides ${ }^{39,71}$. IBA1 staining area to determine microglia density was identified with thresholding based on the background variance. Individual Ibal-stained microglial cells were identified with thresholding after successive edge-preserving smoothing to remove stray processes, and the microglia cell shape was traced by enhancing microglia process shapes and thresholding the resulting image. Plaques were identified with thresholding based on the background variance. Microglial infiltration of plaques was determined from the total Iba1 staining area overlapping plaques for a sample, normalized by total plaque area. Microglial compactness was calculated on individual Iba1-stained cells as a measurement output defined by Cellprofiler as the mean squared distance of the object's pixels from the centroid divided by the area. Calculated this way, this metric is lower for more compact cells; in Fig. 8, we invert this value so that our modules positively correlate with compactness.

Reporting summary. Further information on research design is available in the Nature Research Reporting Summary linked to this article.

\section{Data availability}

The RNA-seq data generated in this study have been deposited in the synapse.org (Sage Bionetworks) database under https://doi.org/10.7303/syn21898410 [https://www.synapse.org/\#! Synapse:syn21898410/wiki/603968]. The RNA-seq data and associated metadata are available under restricted access due to the fact that this data is derived from human tissue samples, and access can be obtained by making a formal request through the synapse.org database. Data from all figures is available in Supplementary Data and Source data files. ROSMAP data ${ }^{1}$ and MSSM data ${ }^{4}$ were both downloaded from synapse.org (Sage Bionetworks) database. ROSMAP data (Synapse ID: syn21589959) was downloaded from https://www.synapse.org/\#! Synapse:syn21589959 and MSSM data (Synapse ID: syn7416949) was downloaded from https://www.synapse.org/\#!Synapse:syn7416949. Source data are provided with this paper.

Received: 3 January 2020; Accepted: 6 September 2021; Published online: 27 September 2021

\section{References}

1. Mostafavi, S. et al. A molecular network of the aging human brain provides insights into the pathology and cognitive decline of Alzheimer's disease. Nat. Neurosci. 21, 811-819 (2018).

2. Olah, M. et al. A transcriptomic atlas of aged human microglia. Nat. Commun. 9, 539 (2018)

3. Zhang, B. et al. Integrated systems approach identifies genetic nodes and networks in late-onset Alzheimer's disease. Cell 153, 707-720 (2013).

4. Wang, M. et al. The Mount Sinai cohort of large-scale genomic, transcriptomic and proteomic data in Alzheimer's disease. Sci. Data 5, 180185 (2018)

5. Leinonen, V. et al. Amyloid and tau proteins in cortical brain biopsy and Alzheimer's disease. Ann. Neurol. 68, 446-453 (2010). 
6. Luikku, A. J. et al. Predicting development of Alzheimer's disease in patients with shunted idiopathic normal pressure hydrocephalus. J. Alzheimers Dis. 71, 1233-1243 (2019).

7. McGovern, R. A. et al. Predicting cognitive improvement in normal pressure hydrocephalus patients using preoperative neuropsychological testing and cerebrospinal fluid biomarkers. Neurosurgery 85, E662-E669 (2019).

8. Hamilton, R. et al. Lack of shunt response in suspected idiopathic normal pressure hydrocephalus with Alzheimer disease pathology. Ann. Neurol. 68, 535-540 (2010).

9. Borgesen, S. E. \& Gjerris, F. The predictive value of conductance to outflow of CSF in normal pressure hydrocephalus. Brain 105, 65-86 (1982).

10. Symon, L. \& Dorsch, N. W. Use of long-term intracranial pressure measurement to assess hydrocephalic patients prior to shunt surgery. $J$. Neurosurg. 42, 258-273 (1975).

11. Espay, A. J. et al. Deconstructing normal pressure hydrocephalus: ventriculomegaly as early sign of neurodegeneration. Ann. Neurol. 82, 503-513 (2017).

12. Leinonen, V. et al. Cortical brain biopsy in long-term prognostication of 468 patients with possible normal pressure hydrocephalus. Neurodegener. Dis. 10, 166-169 (2012).

13. Patel, S. et al. Phosphorylated tau/amyloid beta 1-42 ratio in ventricular cerebrospinal fluid reflects outcome in idiopathic normal pressure hydrocephalus. Fluids Barriers CNS 9, 7 (2012).

14. Libard, S. \& Alafuzoff, I. Alzheimer's disease neuropathological change and loss of matrix/neuropil in patients with idiopathic normal pressure hydrocephalus, a model of Alzheimer's disease. Acta Neuropathol. Commun. 7, 98 (2019).

15. Barnes, J. et al. Alzheimer's disease first symptoms are age dependent: evidence from the NACC dataset. Alzheimers Dement. 11, 1349-1357 (2015).

16. Knopman, D. S. et al. Neuropathology of cognitively normal elderly. J. Neuropathol. Exp. Neurol. 62, 1087-1095 (2003).

17. Schmitt, F. A. et al. Preclinical AD revisited: neuropathology of cognitively normal older adults. Neurology 55, 370-376 (2000).

18. Xuereb, J. H. et al. Neuropathological findings in the very old. Results from the first 101 brains of a population-based longitudinal study of dementing disorders. Ann. NY Acad. Sci. 903, 490-496 (2000).

19. Zhou, Y. et al. Human and mouse single-nucleus transcriptomics reveal TREM2-dependent and TREM2-independent cellular responses in Alzheimer's disease. Nat. Med. 26, 131-142 (2020).

20. Olah, M. et al. Single cell RNA sequencing of human microglia uncovers a subset associated with Alzheimer's disease. Nat. Commun. 11, 6129 (2020).

21. Mathys, H. et al. Single-cell transcriptomic analysis of Alzheimer's disease. Nature 570, 332-337 (2019).

22. Grubman, A. et al. A single-cell atlas of entorhinal cortex from individuals with Alzheimer's disease reveals cell-type-specific gene expression regulation. Nat. Neurosci. 22, 2087-2097 (2019).

23. Leek, J. T. \& Storey, J. D. Capturing heterogeneity in gene expression studies by surrogate variable analysis. PLoS Genet. 3, 1724-1735 (2007).

24. Brouwers, N. et al. Alzheimer risk associated with a copy number variation in the complement receptor 1 increasing C3b/C4b binding sites. Mol. Psychiatry 17, 223-233 (2012).

25. Jansen, I. E. et al. Genome-wide meta-analysis identifies new loci and functional pathways influencing Alzheimer's disease risk. Nat. Genet. 51, 404-413 (2019).

26. Zhang, Y. et al. An RNA-sequencing transcriptome and splicing database of glia, neurons, and vascular cells of the cerebral cortex. J. Neurosci. 34, 11929-11947 (2014).

27. Habib, N. et al. Disease-associated astrocytes in Alzheimer's disease and aging. Nat. Neurosci. 23, 701-706 (2020).

28. Cain, A. et al. Multi-cellular communities are perturbed in the aging human brain and with Alzheimer's disease. Preprint at bioRxiv https://doi.org/ 10.1101/2020.12.22.424084 (2020).

29. Langfelder, P. \& Horvath, S. WGCNA: an R package for weighted correlation network analysis. BMC Bioinformatics 9, 559 (2008).

30. El Gaamouch, F., Jing, P., Xia, J. \& Cai, D. Alzheimer's disease risk genes and lipid regulators. J. Alzheimers Dis. 53, 15-29 (2016).

31. Wong, M. W. et al. Dysregulation of lipids in Alzheimer's disease and their role as potential biomarkers. Alzheimers Dement. 13, 810-827 (2017)

32. Jones, L. et al. Genetic evidence implicates the immune system and cholesterol metabolism in the aetiology of Alzheimer's disease. PLOS ONE 5, e13950 (2010).

33. Wingo, T. S. et al. Association of early-onset Alzheimer disease with elevated low-density lipoprotein cholesterol levels and rare genetic coding variants of APOB. JAMA Neurol. 76, 809-817 (2019).

34. Baez, E. et al. Protection by neuroglobin expression in brain pathologies. Front. Neurol. 7, 146 (2016).

35. Sun, F., Mao, X., Xie, L., Greenberg, D. A. \& Jin, K. Neuroglobin protein is upregulated in Alzheimer's disease. J. Alzheimers Dis. 36, 659-663 (2013).
36. Conway, M., Nafar, F., Straka, T. \& Mearow, K. Modulation of amyloid-beta protein precursor expression by HspB1. J. Alzheimers Dis. 42, 435-450 (2014).

37. Keren-Shaul, H. et al. A unique microglia type associated with restricting development of Alzheimer's disease. Cell 169, 1276.e17-1290.e17 (2017).

38. Mathys, $H$. et al. Temporal tracking of microglia activation in neurodegeneration at single-cell resolution. Cell Rep. 21, 366-380 (2017).

39. Patrick, E. et al. A cortical immune network map identifies distinct microglia transcriptional programs associated with beta-amyloid and tau pathologies. Transl. Psychiatry 11, 50 (2021).

40. Thrupp, N. et al. Single-nucleus RNA-seq is not suitable for detection of microglial activation genes in humans. Cell Rep. 32, 108189 (2020).

41. Alsema, A. M. et al. Profiling microglia from Alzheimer's disease donors and non-demented elderly in acute human postmortem cortical tissue. Front. Mol. Neurosci. 13, 134 (2020).

42. Mancuso, R. et al. Stem-cell-derived human microglia transplanted in mouse brain to study human disease. Nat. Neurosci. 22, 2111-2116 (2019).

43. Hasselmann, J. et al. Development of a chimeric model to study and manipulate human microglia in vivo. Neuron 103, 1016.e10-1033.e10 (2019).

44. Serrano-Pozo, A. et al. Reactive glia not only associates with plaques but also parallels tangles in Alzheimer's disease. Am. J. Pathol. 179, 1373-1384 (2011)

45. Montine, T. J. et al. National Institute on Aging-Alzheimer's Association guidelines for the neuropathologic assessment of Alzheimer's disease: a practical approach. Acta Neuropathol. 123, 1-11 (2012).

46. Reisberg, B., Ferris, S. H., de Leon, M. J. \& Crook, T. The Global Deterioration Scale for assessment of primary degenerative dementia. Am. J. Psychiatry 139, 1136-1139 (1982).

47. Yao, T., Sweeney, E., Nagorski, J., Shulman, J. M. \& Allen, G. I. Quantifying cognitive resilience in Alzheimer's disease: The Alzheimer's Disease Cognitive Resilience Score. PLoS ONE 15, e0241707 (2020).

48. Maarouf, C. L. et al. Alzheimer's disease and non-demented high pathology control nonagenarians: comparing and contrasting the biochemistry of cognitively successful aging. PLoS ONE 6, e27291 (2011).

49. Villemagne, V. L. et al. Longitudinal assessment of Abeta and cognition in aging and Alzheimer disease. Ann. Neurol. 69, 181-192 (2011).

50. Oakley, $\mathrm{H}$. et al. Intraneuronal beta-amyloid aggregates, neurodegeneration, and neuron loss in transgenic mice with five familial Alzheimer's disease mutations: potential factors in amyloid plaque formation. J. Neurosci. 26, 10129-10140 (2006)

51. Cruz, J. C., Tseng, H. C., Goldman, J. A., Shih, H. \& Tsai, L. H. Aberrant Cdk5 activation by $\mathrm{p} 25$ triggers pathological events leading to neurodegeneration and neurofibrillary tangles. Neuron 40, 471-483 (2003).

52. Cruz, J. C. et al. p25/cyclin-dependent kinase 5 induces production and intraneuronal accumulation of amyloid beta in vivo. J. Neurosci. 26, 10536-10541 (2006)

53. Schneider, J. A., Arvanitakis, Z., Bang, W. \& Bennett, D. A. Mixed brain pathologies account for most dementia cases in community-dwelling older persons. Neurology 69, 2197-2204 (2007)

54. James, B. D. et al. TDP-43 stage, mixed pathologies, and clinical Alzheimer'stype dementia. Brain 139, 2983-2993 (2016).

55. Barnes, L. L. et al. Mixed pathology is more likely in black than white decedents with Alzheimer dementia. Neurology 85, 528-534 (2015).

56. Hakim, S. \& Adams, R. D. The special clinical problem of symptomatic hydrocephalus with normal cerebrospinal fluid pressure. Observations on cerebrospinal fluid hydrodynamics. J. Neurol. Sci. 2, 307-327 (1965).

57. Adams, R. D., Fisher, C. M., Hakim, S., Ojemann, R. G. \& Sweet, W. H. Symptomatic occult hydrocephalus with "normal" cerebrospinal-fluid pressure. A treatable syndrome. N. Engl. J. Med. 273, 117-126 (1965).

58. Daou, B., Klinge, P., Tjoumakaris, S., Rosenwasser, R. H. \& Jabbour, P. Revisiting secondary normal pressure hydrocephalus: does it exist? A review. Neurosurg. Focus 41, E6 (2016).

59. Oliveira, L. M., Nitrini, R. \& Roman, G. C. Normal-pressure hydrocephalus: a critical review. Dement. Neuropsychol. 13, 133-143 (2019).

60. Relkin, N., Marmarou, A., Klinge, P., Bergsneider, M. \& Black, P. M. Diagnosing idiopathic normal-pressure hydrocephalus. Neurosurgery 57, S4-S16 (2005).

61. Bret, P., Guyotat, J. \& Chazal, J. Is normal pressure hydrocephalus a valid concept in 2002? A reappraisal in five questions and proposal for a new designation of the syndrome as "chronic hydrocephalus". J. Neurol. Neurosurg. Psychiatry 73, 9-12 (2002).

62. Braak, H., Alafuzoff, I., Arzberger, T., Kretzschmar, H. \& Del Tredici, K. Staging of Alzheimer disease-associated neurofibrillary pathology using paraffin sections and immunocytochemistry. Acta Neuropathol. 112, 389-404 (2006).

63. Thal, D. R., Rub, U., Orantes, M. \& Braak, H. Phases of A beta-deposition in the human brain and its relevance for the development of AD. Neurology 58, 1791-1800 (2002).

64. Andrews, S. FastQC: a quality control tool for high throughput sequence data. https://www.bioinformatics.babraham.ac.uk/projects/fastqc/ (2021). 
65. Dobin, A. et al. STAR: ultrafast universal RNA-seq aligner. Bioinformatics 29, 15-21 (2013).

66. Liao, Y., Smyth, G. K. \& Shi, W. featureCounts: an efficient general purpose program for assigning sequence reads to genomic features. Bioinformatics 30, 923-930 (2014).

67. Love, M. I., Huber, W. \& Anders, S. Moderated estimation of fold change and dispersion for RNA-seq data with DESeq2. Genome Biol. 15, 550 (2014).

68. Durbin, B. P., Hardin, J. S., Hawkins, D. M. \& Rocke, D. M. A variancestabilizing transformation for gene-expression microarray data. Bioinformatics 18, S105-S110 (2002).

69. Ritchie, M. E. et al. limma powers differential expression analyses for RNAsequencing and microarray studies. Nucleic Acids Res. 43, e47 (2015).

70. Kamburov, A., Stelzl, U., Lehrach, H. \& Herwig, R. The

ConsensusPathDB interaction database: 2013 update. Nucleic Acids Res. 41, D793-D800 (2013).

71. Kamentsky, L. et al. Improved structure, function and compatibility for CellProfiler: modular high-throughput image analysis software. Bioinformatics 27, 1179-1180 (2011).

\section{Acknowledgements}

This work was supported by NIH grants K08-AG049938 (to A.F.T.) and K76-AG054868 (to A.F.T.) and with support from the Thompson Family Foundation. We would also like to acknowledge Richard Hickman for assistance with developing the tau grading system.

\section{Author contributions}

W.H., X.F., S.L., D.B., E.S., V.M., S.M., E.H.H.Y. and Z.T. all contributed to organizing and analyzing the data in this manuscript. A.M.B. and H.X. assisted with completion and analysis of immunofluorescence experiments. G.M.M. recruited the patients, evaluated them, operated on them, and made sure the tissue was acquired properly and in a timely fashion, and was assisted by G.I. and R.A.M. A.F.T. designed the study and subsequent analyses, oversaw its completion, and wrote the manuscript together with W.H.

\section{Competing interests}

The authors declare no competing interests.

\section{Additional information}

Supplementary information The online version contains supplementary material available at https://doi.org/10.1038/s41467-021-25902-y.

Correspondence and requests for materials should be addressed to Andrew F. Teich.

Peer review information Nature Communications thanks Charlotte Teunissen, Renzo Mancuso, and the other anonymous reviewer(s) for their contribution to the peer review of this work

Reprints and permission information is available at http://www.nature.com/reprints

Publisher's note Springer Nature remains neutral with regard to jurisdictional claims in published maps and institutional affiliations.
Open Access This article is licensed under a Creative Commons Attribution 4.0 International License, which permits use, sharing, adaptation, distribution and reproduction in any medium or format, as long as you give appropriate credit to the original author(s) and the source, provide a link to the Creative Commons license, and indicate if changes were made. The images or other third party material in this article are included in the article's Creative Commons license, unless indicated otherwise in a credit line to the material. If material is not included in the article's Creative Commons license and your intended use is not permitted by statutory regulation or exceeds the permitted use, you will need to obtain permission directly from the copyright holder. To view a copy of this license, visit http://creativecommons.org/ licenses/by/4.0/.

(C) The Author(s) 2021 